\title{
TRIB-04-1047
}

\section{A Finite Element Study of the Residual Stress and Deformation in Hemispherical Contacts}

\author{
by \\ Robert Jackson (Member, ASME) ${ }^{1}$ \\ Itti Chusoipin \\ Itzhak Green (Fellow, ASME) \\ George W. Woodruff School of Mechanical Engineering \\ Georgia Institute of Technology \\ Atlanta, GA 30332-0405
}

\begin{abstract}
This work presents a finite element model (FEM) of the residual stresses and strains that are formed after an elasto-plastic hemispherical contact is unloaded. The material is modeled as elastic perfectly-plastic and follows the von Mises yield criterion. The FEM produces contours for the normalized axial and radial displacements as functions of the removed interference depth and location on the surface of the hemisphere. Contour plots of the von Mises stress and other stress components are also presented to show the formation of the residual stress distribution with increasing plastic deformation. This work shows that high residual von Mises stresses appear in the material pile-up near the edge of the contact area after complete unloading. Values are defined for the minimum normalized interference, that when removed, results in plastic residual stresses. This work also defines an interference at which the maximum residual stress transitions from a location below the contact region and along the axis of symmetry to one near to the surface at the edge of the contact radius (within the pile-up).
\end{abstract}

\footnotetext{
${ }^{1}$ Currently at: Department of Mechanical Engineering, Auburn University, Auburn, AL 36849-5341 (robert.jackson@eng.auburn.edu).
} 


\section{Nomenclature}

$C=$ critical yield stress coefficient

$E=$ elastic modulus

$P=$ contact force

$R=$ radius of hemispherical asperity

$S_{y}=$ yield strength

$a=$ contact radius

$r=$ radial distance from axis of symmetry

$\gamma=$ radial displacement

$\omega=$ interference between hemisphere and surface

$v=$ Poisson's ratio

\section{Subscripts}

$c=$ critical value at onset of plastic deformation

$o=$ original

$v m=$ von Mises stress

\section{Superscripts}

'= equivalent or displaced

$*=$ normalized 


\section{Introduction}

The case of an elasto-plastic hemispherical contact with a rigid plane has important engineering applications in both the macro and micro scale. The current model is normalized to be valid in both scales (i.e., the hemispherical radius, $R$, can assume any value, and as long as the material can still be modeled as a continuum). It has been well established that asperities will deform plastically during the contact of rough surfaces. It is also clear that in many applications the load will periodically be removed or cycled. This action makes it desirable to know the effect the contact has had on the surface material and the geometry through plastic deformations and residual stresses. Such information may be useful in analyzing the friction, wear and deformation of contacts, as for example, in micro-switches, boundary lubrication, rolling element bearings, metal forming, fretting, and shot peening.

Jackson and Green [1], Kogut and Etsion [2], and Mesarovic and Fleck [3] provide results for the loaded condition case. As a continuation of these previous results, the current work is focused on the residual stress and deformation, which remain after the interference has been removed (see Fig. 1). The model by Jackson and Green [1] is regenerated to simulate the loaded condition and the unloaded condition for the first time. The von Mises yield criteria is used to indicate whether the hemisphere material is deformed elastically or plastically. The material is assumed to act elastic perfectly-plastic, so that there is no strain hardening effect. Experimentally, Johnson [4] observed the contact of bronze and steel spheres pressed against a steel flat. In order to make measurements of the deformation, he also unloaded the spheres. Once unloaded, he observed permanent indentation of both the sphere and the flat surface, along with a pileup or crown of raised material around the contact area. These findings 
match those found through FEM simulation in this work. Tabor [5] also recognizes the need to consider these effects when measuring the hardness of a surface using an indentation test.

Kral et al. [6-8] modeled the inverse case of a repeated elasto-plastic contact of the rigid sphere against a non-layered and layered half-space using FEM. Although based on a different case, their model produces qualitatively similar results to the one presented here. While Kral et al. applies a load up to 300 times the initial load to cause yielding (critical load), the current work more than doubles this value by modeling a load of 750 times the critical load. Practical experience indicates that in applications such as shot peening, EHD and other forms of contact, large amounts of deformation can occur far into the elasto-plastic regime. In the asperity contact between rough surfaces, some very high asperities or peaks are likely to be heavily loaded.

Ye and Komvopoulos [9] also simulate the contact in a layered deforming half space and a rigid sphere, although they manually apply a hydrostatic residual stress prior to contact. These applied residual stresses model surface treatments such as shot peening. They then attempt to quantify the effect of the applied residual stresses on the contact deformation and stresses. In addition, they also investigate the effect of sliding on the resulting stresses. Despite these works and other previous works, there is currently no in depth analysis of the residual stresses and deformations of an unloaded elasto-plastic spherical contact against a rigid flat.

In the previous model by Jackson and Green [1], the model was simulated under the loaded conditions for many interferences and five steel materials, during which the hemisphere deforms in the elastic, elasto-plastic, and fully plastic regimes. The following definitions are given for the regimes: 1) the elastic regime considers deformation absent of plasticity, 2) the elasto-plastic regime contains plastically deformed material but the contact area still contains an elastic region, and 3) the fully plastic regime defines the case of a contact whose area of normal 
pressure yields entirely. The measurement of hardness requires that the contact reaches the fully plastic regime, where the average contact pressure has traditionally been regarded as the hardness. However, the hardness should not be implemented as a material property, as it also varies with deformation, geometry, and material properties such as yield strength, Poisson's ratio and the elastic modulus (see Jackson and Green [1]). The nomenclature here conforms with the said work.

This work defines the interference depth, $\omega$, as the distance the original hemisphere shape is pressed into the rigid flat (see Fig. 1). The normalized interference depth, $\omega^{*}$, is defined as

$$
\omega^{*}=\frac{\omega}{\omega_{c}}
$$

where, $\omega_{\mathrm{c}}$ is the critical interference and is given by Jackson and Green [1] as

$$
\omega_{c}=\left(\frac{\pi C S_{y}}{2 E^{\prime}}\right)^{2} R
$$

The corresponding critical contact radius is

$$
a_{c}=\sqrt{\omega_{c} R}
$$

where, $C=1.639, S_{\mathrm{y}}$ is the yield stress, and $E$ ' is the equivalent elastic modulus, and $R$ is the equivalent radius. When $\omega^{*}<1$ then the hemisphere deforms in elastic regime. When $\omega^{*}>1$ the 
deformation is in elasto-plastic regime. At approximately $70 \leq \omega^{*} \leq 110$, the deformation reaches the fully plastic regime (Jackson and Green [1], Kogut and Etsion [2]). For residual stresses and strains to remain once the hemisphere is unloaded, a $\omega^{*}$ greater than one must be applied (see diagram in Fig. 1). The critical contact radius, $a_{c}$, defines the radius of the area of contact at an interference depth of $\omega_{\mathrm{c}}$. From this point forward residual stress and residual displacement will refer to stress and displacement that remain in the elasto-plastic hemisphere after the load is completely removed.

\section{Finite Element Model}

There are two ways to simulate this contact model. In the first approach, the force is applied to the hemisphere and then the displacement is computed. In the second approach an interference, $\omega$, is applied and the contact force is calculated. In this work, the second approach is used because the solution converges more rapidly than the first one. The contact forces are determined by summing the reaction forces on the base nodes of the hemisphere.

The finite element solution is generated by the Ansys ${ }^{\mathrm{TM}}$ software packages. To increase the efficiency, a 2-D axisymmetric model is used. Several mesh refinements have been performed to reduce the errors in the residual stresses (See Fig. 2 for example mesh). For this investigation ANSYS element types Plane 82, Contact 169, and Contact 172 are used. The fine area of the mesh near the tip of the hemisphere is varied in order to encompass the region of high stress near the contact. The mesh is constructed using eight node solid elements and 100 contact elements at the area of contact. The meshed contact area is also varied to ensure that at least 30 contact elements are in contact for each applied interference (maximum contact radius error of 
3.3\%). The resulting mesh consists of over 11,101 elements. The mesh has extensively been verified for model convergence by Jackson and Green [1] and Quicksall et al. [10].

As shown in Fig. 3 constraints in the $x$ and $y$ directions were applied to the nodes on the base, while a radial constraint is applied to the symmetric axis. This boundary condition may be valid for the modeling of asperity contacts for two reasons: (1) The asperities are actually connected to a much larger bulk material at the base and will be significantly restrained there, and (2) Since the high stress region occurs near the contact, the boundary condition at the base of the hemisphere will not greatly effect the solution because of Saint Venant's Principle. On sample problems given in Ref. [1], the change in results between the said boundary conditions and one in which the nodes along axis $\mathrm{x}$ are allowed to translate radially, have shown only marginal difference (less than 3\% difference in area, and less than $1 \%$ in load). In principal, however, when large deformations are imposed, boundary conditions may significantly influence the results. Also in this case, the rigid contact line is constrained in the $x$ (radial) direction, while the interference, $\omega$, is applied as a displacement in the $y$ (axial) direction.

A large range of interferences are applied to the FEM model and then the contact force, stress tensor, von Mises stresses and the displacement in both the radial and axial directions are recorded. After the loaded condition has been simulated (giving the same results as in Jackson and Green [1]) the solution is then restarted and unloaded completely to simulate the residual stresses and the displacements. Since the problem is nonlinear, small load steps are used to increment toward a solution in both loading and unloading. 


\section{Results and Discussion}

The results are presented for a range of normalized interferences, $\omega^{*}$, from 0.571 to 171 . The material properties used are for a steel material (extracted from [11]) and presented in Table 1. These material properties allow for effective modeling of all the elasto-plastic contact regimes. The computation time is about an hour for small interferences and two to three hours for large interferences on a $3.2 \mathrm{GHz}$ PC.

As an additional check of the model's validity, the contact forces during the unloaded conditions are calculated. Based on the force balance solution, once the contact is completely unloaded the reaction force should be identically zero. This trivial condition is consistently satisfied with an eight-node FEM model which computes the reaction force to be about ten orders of magnitude smaller than the load originally applied to the hemisphere.

\section{Displacement}

The axial and radial surface displacements of the nodes on the hemisphere surface are monitored in order to investigate the deformation of the hemisphere. As shown in Fig. 3 the axial and radial directions correspond to the $y$-axis and $x$-axis, respectively. While $\omega_{c}$ effectively normalized the axial displacement, $U_{y}$, it is ineffective in normalizing the radial displacement,

$U_{x}$. It is found (see the Appendix) that $U_{x}$ is effectively normalized by $\gamma_{c}=\frac{1}{6} \frac{\left(\omega_{c}\right)^{\frac{3}{2}}}{\sqrt{R}}$ which is the relative radial displacement of the critical contact radius before and after loading. In this section plots of the normalized axial and radial displacements, $U_{x} / \gamma_{c}$ and $U_{y} / \omega_{c}$, with respect to the normalized radial distance, $r / a_{c}$, are presented for both the loaded and unloaded conditions (see Figs. 4-7). Note that $r$ is the radial distance from the axis of symmetry (y-axis) to a point on the 
surface. Thus, $r$ is analogous to the $x$-coordinate of a location on the hemisphere surface. The displacements are presented relative to the hemisphere surface, such that curvature is mitigated. Although the main focus of this work is the unloaded case, the surface displacements for the loaded case are also presented. Although, Fischer-Cripps [12] has provided results for the purely elastic case of Hertz contact.

The current results have also been compared to the analytical predictions of Kogut and Etsion's [13] given for the separation between the deformed sphere and the rigid flat. The results are compared at benchmark values of $\omega^{*}=4.29$ and $\omega^{*}=100$ (near the benchmark values of $\omega^{*}=4$ and $\omega^{*}=110$ used in their work). When the deformation is nearly elastic at $\omega^{*}=4.29$, the results are almost exactly equivalent until approximately $r / a_{c}=5$. Past this value the results differ significantly. For the elasto-plastic deformation at $\omega^{*}=100$, the results differ significantly after $r / a_{c}$ increases past a value of approximately 14 . The reason for these differences is likely because Kogut and Etsion's equations are based on a perfectly elastic contact solution given in Muller et al. [14].

\section{Loaded Displacement}

Figures 4 and 5 show the surface displacement in both the axial $\left(U_{y} / \omega_{c}\right)$ and radial $\left(U_{x} / \gamma_{c}\right)$ direction for the loaded hemisphere. These plots show the evolution of hemisphere surface deformation with increasing interferences. As expected, the displacements increase with the normalized interference depth. The boundary between the contact region and the free boundary of the hemisphere can be clearly seen through the sharp changes of the slope in the radial displacement plot (see Fig. 4). In the low interferences, the surface displaces radially in mostly the negative direction. This is because at the small normalized interferences most of the 
material in the hemisphere is deforming elastically and allowed to compress. As the interference significantly increases past the critical interference, the hemisphere increasingly deforms plastically and the material in the contact region increasingly displaces outward into the positive $x$ direction. This bulging occurs because as the deformation increases, yielded material flows plastically, and is assumed incompressible abiding by a Poisson's ratio effectively equal to 0.5 [15].

\section{Unloaded Displacement}

In this section the unloaded or residual displacement along both the radial and axial direction $\left(U_{x} / \gamma_{c}\right.$ and $\left.U_{y} / \omega_{c}\right)$ of the hemisphere are monitored with respect to the normalized radial distance, $r / a_{c}$ (see Figs. 6 and 7). The residual displacement is defined here as the displacement on the surface which remains after the hemisphere is completely unloaded from a normalized penetration depth, $\omega^{*}$. The residual displacements occur when the hemisphere has plastically deformed and does not fully recover to its original shape (see schematic in Fig. 1). The displacements are also labeled for each normalized penetration depth, $\omega^{*}$, from which the hemisphere is unloaded.

As seen from the normalized residual displacement plots (Figs. 6 and 7), once the hemisphere is loaded to $\omega^{*}>1$ (which marks the transition from the elastic to elasto-plastic regime) and then unloaded, the residual displacements tend to increase with the magnitude of the removed load (see Fig. 6). Comparing Figs. 4(a) and 6(a), at small normalized interferences the trends between the loaded and unloaded cases are very different. After a small normalized interference is removed, the hemisphere is still mostly elastic, with only a small region of plastic deformation. Most of the hemisphere material then tries to restore its original shape, while only 
a small portion resists. In the radial and axial direction this results in regions of negative and positive deformation when the hemisphere is unloaded. The negative deformation occurs above the plastic core, while the positive deformation occurs mostly outside of this region. This phenomenon is known as a residual pile-up, which is further enhanced for larger deformations. The curvature of the hemisphere has the effect of negating the material pile-up so that the unloaded hemisphere is essentially flattened, resulting in "out-of-roundness" for the hemisphere. A dimple or indentation will form on a surface with little curvature.

After large interferences are removed, the plastic regions dominate, and the material remains more in the plastically deformed geometry (see Figs. 6(b) and 7(b)). In contrast to smaller residual deformations (Figs. 6(a) and 7(a)), the residual surface displacements after large loads mimic the deformations of the loaded hemisphere (see Figs 4(b) and 5(b)). However, there are some regions which still remain elastic and tend to return to their undeformed shape when unloaded. Therefore, the overall magnitudes of the residual displacements are less than that of the loaded conditions. Also, the residual displacements in the axial direction tend to change direction when unloaded and cause a crown of material to rise around the unloaded contact region (see Fig. 7). This occurs near the edge of the contact area and is referred to as the previously mentioned residual pile-up. The peaks of deformation in both the $x$ and $y$ direction are located at the same $r / a_{c}$ and correspond to the residual pile-up. As the load that the hemisphere is unloaded from increases, the pile-up acquires a sharper edge.

The residual pile-up marks the sharp transition from the contact region to the free boundary and it also increases in magnitude with the normalized interferences from which the hemisphere is unloaded. Kral et al. [6-8] and Ye and Komvopoulos [9] also confirm the occurrence of pile-up during the FEM analysis of the repeated indentation of a half-space by a 
rigid sphere. Johnson [4] also experimentally confirms the existence of a residual pile-up. Residual pile-up readily occurs during indentation type hardness tests after unloading, and must be accounted for when making hardness measurements [5].

These deformations change the surface profile of the hemisphere. Also, the contact of the asperities on rough surfaces is commonly modeled by hemispherical contact. This indicates that the surface topographies of heavily loaded rough surfaces will also change after the load is removed. The current analysis suggests that after a rough surface is unloaded from plastic deformation, the surface asperities will be flattened and have a pile-up region around each contact. If the asperity has a large radius of curvature in relation to the contact radius, the pile-up may also cause an indentation in the surface. These changes in topography are important in such cases as boundary lubrication and sliding friction. The changes in the surface profile will also affect heavily loaded ball bearings. For ball bearings to operate properly, the balls must be as close as possible to spherical in shape. This work shows that after unloading from heavy loads, the balls may lose their original spherical shape and have the potential to cause bearing failure.

\section{Stress Formation}

Since the stresses of loaded spherical contacts deforming elastically and plastically are well documented, they will not be presented in detail here. Extensive analyses of the stress evolution in loaded hemispheres are given in Johnson [16], Jackson and Green [1], and Kogut and Etsion [2]. However, in order to understand the residual stress evolution it is important to understand how the stresses originally developed during the loading of the hemisphere. For this reason a brief summary of the stress evolution during loading is given next. 
At low interferences a high stress region starts to form below the contact interface. Eventually the material yields in this high stress region and a plastic core forms. The plastic core is surrounded by elastic material, which diminishes as the hemisphere is subjected to larger interferences. At higher interferences the plastic core expands in a 3-D fashion to the surface, and also inward toward the center of the hemisphere. The reason the plastic region expands is because the material in the hemisphere that is flowing plastically can no longer resist additional load. Therefore, any additional load is carried by the surrounding elastic regions. At about $\omega^{*}=8$ the plastic core reaches the surface near the edge of contact (see [1]). Then there is an elastic core below the contact area that is surrounded by plastically deforming material. At a much higher load, anywhere within $70 \leq \omega^{*} \leq 110$ (depending on the material properties), the plastic region covers the entire contact area and occupies a large portion of the space in the hemisphere. This is known as the fully plastic regime. As mentioned earlier, the average contact pressure in the fully plastic regime is traditionally defined as the hardness. The hardness, however, is not a material property and varies with the undeformed and deformed geometry as predicted by Tabor [5] and numerically verified by Jackson and Green [1].

\section{Residual Stress Formation}

The value of the von Mises stress is used in this analysis to predict yielding of the hemisphere material. However, by calculating the von Mises stress some information about the material stress tensor is lost. The von Mises stress shows how 'intense' the stress state is relative to the yield strength; However, as a positive quantity it does not reveal if the material is in tension, compression, or under shear etc. In the study of crack initiation and propagation it is important to know the orientation of stress in relation to a crack and if stress is tensile or 
compressive [17]. Thus it is important to first understand the distribution of the complete stress tensor throughout the hemisphere.

When the plastically deformed hemisphere is unloaded, the elastic material attempts to restore its original shape. However, the plastically deformed regions inhibit this since the material 'memory' or 'state' has changed. This results in regions of tension and compression, even though the overall force applied to the system sums to zero. The plots of the 3-D stress tensor $\left(\sigma_{x}, \sigma_{y}, \sigma_{z}, \tau_{x y}\right)$ for a hemisphere unloaded from $\omega^{*}=3.92$ show clearly these regions of tension and compression (see Fig. 8). Since the problem is axisymmetric the shear stresses $\tau_{x z}$ and $\tau_{y z}$ are identically zero. These results are also given for a hemisphere unloaded from $\omega^{*=35}$ in Fig. 9.

The distribution of $\sigma_{x}$ shows compressive and tensile radial stress regions. Figure 8(b) shows the interesting distribution of stresses in the $y$ direction. Near the plastic core $\sigma_{y}$ is tensile and $\sigma_{x}$ is compressive. For $\sigma_{y}$ there is a band of compressive stresses below the edge of contact and also along the axis of symmetry but closer to the center of the hemisphere. The differing stress distributions of $\sigma_{x}$ and $\sigma_{y}$ will contribute to larger von Mises stresses in certain regions. For instance, a region will have higher von Mises stresses and be closer to yielding if orthogonal normal stresses differ in sign or magnitude.

Figure 8(c) depicts stress contours for the residual hoop stress, $\sigma_{z}$. If the stress values are followed along the axis of symmetry, it is apparent that it switches between tension and compression several times. As mentioned, this results in complex formation of the von Mises stress.

The contour plot of the residual shear stress $\left(\tau_{x y}\right)$ in Fig. 8(d), for a hemisphere unloaded from $\omega^{*}=3.92$, shows an interesting distribution. Near the edge of unloaded contact, there is a 
region of positive shear stress close to the axis of symmetry that lies next to a region of negative shear stress. The shear stress seems to peak at points away from the axis of symmetry, thus forming hoops of high shear stress around the circumference of the hemisphere. This shear stress amplifies the von Mises stresses within the hemisphere.

The various stress contours which map the complete stress tensor throughout the unloaded hemisphere are also presented for a hemisphere unloaded from a larger interference depth of $\omega^{*}=35.0$ in Fig. 9. In comparison to Fig. 8, these contour plots show how the residual stresses evolve and spread through the hemisphere with increasing plastic deformation. Clearly, the stress distributions can change significantly as load and plastic deformation are increased. Although the residual stresses still exhibit similar regions of tension and compression as shown for $\omega^{*}=3.92$ in Fig. 8 .

Interestingly, in Figs. 9a and 9c there are regions of high tensile stresses in the $x$ and $z$ direction at a point near to the unloaded edge of contact. These stresses correspond to the location of the residual pile-up identified earlier. It seems that when the hemisphere is unloaded, the yielded material, in conjunction with the elastic restoring effect, push the pile-up area upward in the $y$-direction. This action causes tensile stresses in the $x$ and $z$ directions.

Contour plots of the residual von Mises stress (Figs. 10-11) are also generated in order to monitor the intensity of the residual stress formation in the hemisphere. Figure 10 shows purely elastic residual von Mises stress distributions while Fig. 11 shows the onset and formation of plastic regions. The plots display the results for a hemisphere unloaded from a range of $2.14 \leq \omega^{*} \leq 100.0$. As the plastic deformation within the hemisphere increases due to larger interferences and then unloaded, the residual stresses increase and migrate. This migration causes the maximum von Mises stress to move from one location to another. The maximum 
stress location then transitions from a point on the axis of symmetry to a point near the surface at the edge of the unloaded contact area. The maximum stress location after the shift corresponds to the location of the residual pile-up seen in Fig. 7. Table 2 shows the location of the maximum residual stress for hemispheres unloaded from various normalized interferences.

The value $(R-y) / \omega_{c}$ is the normalized depth of maximum von Mises stress from the contacting tip of the hemisphere and $r / a_{c}$ is the normalized distance of the maximum von Mises stress to the $y$-axis. Examining the values of $r / a_{c}$ and $(R-y) / \omega_{c}$ in Table 2, the normalized location of the maximum von Mises stress shifts from the axis of symmetry to the surface for a hemisphere unloaded from normalized interferences between 3.57 and 3.92. This shift signifies the migration of the residual stresses from one location of the plastic core to the residual pile-up at the edge of unloaded contact. However, once the material remains plastic after unloading there is no single point of maximum von Mises stress since regions of plasticity are formed (see Fig. 11). The maximum von Mises stress normalized by the yield stress is plotted in Fig. 12 for hemispheres unloaded from increasing values of $\omega^{*}$. This plot also shows how the maximum von Mises stress levels off to the yield strength for a hemisphere unloaded from a normalize interference within $25 \leq \omega^{*} \leq 30$. This value signifies a threshold that indicates residual plastic stresses. In other words, this value marks the minimum load that when removed, a region in the hemisphere has a high enough residual von Mises stress to remain plastic. Then the region of plastic residual stress grows at the edge of contact when the hemisphere is unloaded from increasing values of normalized penetration depth, $\omega^{*}$ (see Figure 11). The plastic residual stress appears to grow along the surface away from the unloaded area of contact. Since the unloaded hemisphere's location of maximum von Mises stress transitions to the surface, the location of plastic stress in the loaded and unloaded hemisphere do not always correspond. 


\section{Comparison between Aluminum and Steel}

In order to measure the effect of the material properties on the hemisphere deformation, an aluminum hemisphere is also modeled for a hemisphere unloaded from a $\omega^{*}=135$. Table 1 shows the properties used for aluminum as taken from [11]. As previously, the radius, $R$, is held constant.

Figure 13 and 14 show the plot of the normalized axial and radial displacement as a function of the normalized radial distance, $r / a_{c}$, on both the loaded (Fig. 13) and unloaded (Fig. 14) condition and for both materials. An inset is provided for a plot of displacement normalized by the constant hemisphere radius, $R$. From the plots, the deformation of the aluminum and steel hemisphere tend to follow the same trend. However, the values of the displacements normalized by $R$ (or by $\omega_{c}$, which is not shown) are quantitatively very quite different. It appears that the normalizations $\left(U_{x} / \gamma_{c}\right.$ and $\left.U_{y} / \omega_{c}\right)$ used are effective at generalizing the results for the two different materials (see Appendix). As stated in the previous section, the deformation of the hemisphere also depends on the properties of the material as well as the interference. Even though loaded to the same normalized interference, the steel is compressed down with the real displacement of $4.7 \%$ of $R$, while the aluminum is compressed down with only $1.4 \%$ of $R$. Without normalization, the differences in the interference are significantly large, causing the differences in the displacements to also be large. The residual pile-up can still be spotted for both materials, as the contact was loaded to the fully plastic regime.

\section{Repeated Contact}

As elastic perfectly-plastic theory suggests, when an identical repeated load is applied to the hemisphere after being unloaded from elastic perfectly-plastic deformation, the hemisphere 
returns to precisely the same loaded state as the initial loading. FEM results confirm that the deformation returns to exactly the same values with repeated contact of the same load. This occurs because the material undergoes no strain hardening, i.e. the load carrying capacity of the hemisphere material does not change with contact, even though it has plastically deformed. Introducing history dependant strain hardening is expected to alter these results. It should be noted, that in the contact of real rough surfaces in which the asperities do not align, bulk materials deform, and there is slip or sliding, the asperity contacts may not align and behave as described above.

\section{Conclusions}

This work presents a finite element model (FEM) of the residual stresses and strains that are formed after an elasto-plastic hemispherical contact is unloaded. The material is modeled as elastic perfectly-plastic and follows the von Mises yield criterion. A 2-D axisymmetric finite element model of an elastic perfectly-plastic hemisphere in contact with a rigid flat surface is used to calculate the residual stresses and deformations. At even light loads the residual stresses and deformations change the surface geometry of the hemisphere significantly and must be accounted for in cases such as in indentation tests, and rolling element bearings. This affect can also be applied to the repeated contact of rough surfaces when the alignment between them changes between load cycles.

The FEM produces contours for the axial and radial displacements as functions of the removed normalized interference depth and location on the surface of the hemisphere. The displacements are given relative to the surface. The displacements show how the deformation changes from elastic to elasto-plastic as the hemisphere begins to bulge outward instead of 
compress. A material pile-up can clearly be seen in Figure 7b of the residual axial displacement of the hemisphere after it is unloaded. This occurrence is also verified experimentally by Johnson [4] and also by the FEM analysis of Kral et al. [6-8] on the repeated indentation of a half- space by a rigid hemisphere. Still, Kral et al. simulates the contact for about half the range of the current work.

Contour plots of the stress tensor components and the von Mises stress show the development of the residual stress distribution with increasing plastic deformation. This development results in a high stress residual pile-up appearing near the edge of the unloaded contact area. The approximate value for the minimum normalized interference, that when removed, a region of the residual stresses in the hemisphere remains plastic is found to be between $25 \leq \omega^{*} \leq 30$. This work also defines an normalized interference of about $3.57 \leq \omega^{*} \leq 3.92$ at which the maximum residual stress transitions from a location below the contact region and along the axis of symmetry to one near to the surface at the edge of the unloaded contact radius (within the pile-up).

Finally, this work analyzes the effect of material properties on the surface displacements. The deformation of the hemisphere is dependent on the properties of the material and the interferences. With a difference in Young's modulus, Poisson’s ratio, and yield strength, the aluminum tends to deform differently from steel at the same normalized penetration depth. It appears that the normalization used for the displacements is effective at generalizing the results for both sets of material properties, and the given geometry and boundary conditions shown in Fig. 3. 


\section{References}

[1] Jackson, L., Robert, and Green, Itzhak, 2004, “A Finite Element Study of Elasto-Plastic Hemispherical Contact,” Accepted for Publication, ASME J. Tribol.

[2] Kogut, L., \& Etsion, I., 2002, "Elastic-Plastic Contact Analysis of a Sphere and a Rigid Flat," J. of Applied Mechanics, ASME J. Appl. Mech., 69(5), pp. 657-662.

[3] Mesarovic, S. D. and Fleck, N. A., 2000, "Frictionless Indentation of Dissimilar Elastic-plastic Spheres,” Int. J. Solids and Structures, 37, pp.7071-7091.

[4] Johnson, K. L., 1968, "An Experimental Determination of the Contact Stresses Between

Plastically Deformed Cylinders and Spheres,” Engineering Plasticity, Cambridge University

Press, Cambridge, pp. 341-361.

[5] Tabor, D., 1951, The Hardness of Materials, Clarendon Press, Oxford, pp.14-15.

[6] Kral, E. R., Komvopoulos, K., Bogy, D. B., 1993, "Elastic-Plastic Finite Element Analysis of Repeated Indentation of a Half-Space by a Rigid Sphere,” ASME J. Appl. Mech., 60, pp. 829841.

[7] Kral, E. R., Komvopoulos, K., Bogy, D. B., 1995, “Finite Element Analysis of Repeated Indentation of an Elastic-Plastic Layered Medium by a Rigid Sphere, Part I: Surface Results,” ASME J. Appl. Mech., 62, pp. 20-28.

[8] Kral, E. R., Komvopoulos, K., Bogy, D. B., 1995, “Finite Element Analysis of Repeated Indentation of an Elastic-Plastic Layered Medium by a Rigid Sphere, Part II:

Subsurface Results,” ASME J. Appl. Mech., 62, pp. 29-42.

[9] Ye, N., and Komvopoulos, K., 2003, "Effect of Residual Stress in Surface Layer on Contact Deformation of Elastic-Plastic Layered Media,” ASME J. Tribol., 125, pp. 692-699.

[10] Quicksall, J., Jackson, R. L., Green, I. 2004, Elasto-plastic Hemispherical Contact for Varying Mechanical Properties, Accepted to IMechE J. of Eng. Trib. - Part J.

[11] Shigley, J. E., Mischke, C. R., 1989, Mechanical Engineering Design, $5^{\text {th }}$ Edition, McGraw-Hill Inc., New York.

[12] Fischer-Cripps, A. C., 1999, “The Hertzian Contact Surface,” J. Mat. S., 34, pp. 129-137. [13] Kogut, L., Etsion, I., 2003, “Adhesion in Elastic-Plastic Spherical Microcontact,” J. Colloid Interface S., 261, pp. 372-378.

[14] Muller, V. M., Derjaguin, Y.P., Toporov, 1983, “On Two Methods of Calculation of the Force of Sticking of an Elastic Sphere to a Rigid Plane,” Colloids Surf., 7, pp. 251-259. [15] Goodier, J. N., Hodge, P. G., 1958, Elasticity and Plasticity, Wiley \& Sons, Inc., New York.

[16] Johnson, K. L., 1985, Contact Mechanics, Cambridge University Press, Cambridge. [17] Dowling, N. E., 1993, Mechanical Behavior of Materials, Prentice-Hall, Inc., Englewood Cliffs, New Jersey. 


\section{Appendix: Normalization of Displacement}

It is important to find an effective method of normalization for the surface displacements so that the presented results may be applied to a general hemispherical contact with the boundary conditions in Fig. 3, a radius $R$ and material properties $E$, $v$, and $S_{y}$. The vertical displacement $U_{y}$ is effectively normalized by $\omega_{c}$, which is the relative distance that the contact point at the centerline travels before and after loading is applied at the onset of plasticity (Figs. 13(b), 14(b)). A similar typical distance in the radial direction, $\gamma_{c}$, is sought for normalizing $U_{x}$. The quantity $a_{c}$ identifies the radius of the contact at the onset of plasticity. To find out the distance that this point travels radially, its location before loading, $a_{c o}$, is sought such that $\gamma_{c}=a_{c^{-}} a_{c o}$. Finding this quantity results in the normalization

$$
\frac{U_{x}}{\gamma_{c}}=\frac{U_{x}}{a_{c}-a_{c o}}
$$

By assuming no slip occurs between the hemisphere and the rigid flat, $a_{c o}$ is easily approximated. As shown in Fig. 15 the hemisphere surface essentially wraps onto the rigid

surface. This results in the arc $A B$ deforming into the segment $\overline{A^{\prime} B^{\prime}}$, such that $\overline{A^{\prime} B^{\prime}}=A B$. And since $\overline{A^{\prime} B^{\prime}}=a_{c}, A B=a_{c}$. The angle $\theta$ is then calculated as

$$
\theta=\frac{A B}{R}=\frac{a_{c}}{R}
$$

Next, $a_{c o}$ is calculated to be

$$
a_{c o}=R \sin (\theta)=R \sin \left(\frac{a_{c}}{R}\right)
$$

Substituting Eq. (3) into Eq. (A3) and simplifying results in

$$
a_{c o}=R \sin \left(\frac{\sqrt{\omega_{c} R}}{R}\right)=R \sin \left(\sqrt{\frac{\omega_{c}}{R}}\right)
$$


Now $\gamma_{c}$ is defined by

$$
\gamma_{c}=a_{c}-a_{c o}=a_{c}-R \sin \left(\sqrt{\frac{\omega_{c}}{R}}\right)=\sqrt{\omega_{c} R}-R \sin \left(\sqrt{\frac{\omega_{c}}{R}}\right)
$$

Then factoring out $R$ from the right side of the Eq. (A5) gives

$$
\gamma_{c}=R\left[\sqrt{\frac{\omega_{c}}{R}}-\sin \left(\sqrt{\frac{\omega_{c}}{R}}\right)\right]
$$

Letting $x=\sqrt{\frac{\omega_{c}}{R}}$ and using the approximation

$$
\sin x=x-\frac{x^{3}}{3 !}+\frac{x^{5}}{5 !}-\frac{x^{7}}{7 !}+\ldots
$$

results in

$$
x-\sin x \approx \frac{x^{3}}{3 !}
$$

after neglecting higher order terms. Then, Eq. (A6) is approximated using Eq. (A8).

$$
\gamma_{c} \approx R\left[\frac{1}{6}\left(\frac{\omega_{c}}{R}\right)^{\frac{3}{2}}\right]=\frac{1}{6} \frac{\left(\omega_{c}\right)^{\frac{3}{2}}}{\sqrt{R}}
$$

Equation (A9) is tried as an effective normalization of $U_{x}$ by $\gamma_{c}$. The results of steel and aluminum hemispheres loaded and unloaded from $\omega^{*}=135$ are presented in Figs. 13 and 14. It appears from the plots that the normalizations derived are effective to some degree at generalizing the problem for the two different sets of material properties. The ratio of $\gamma_{c} / \omega_{c}$ is also presented in Table 1 for both materials. For both materials the value of $\gamma_{c}$ is much smaller than $\omega_{c}$. 


\section{Tables and Figures}

Table 1: The material properties and critical interferences for Aluminum and Steel

\begin{tabular}{|l|l|l|l|l|l|}
\hline Material & $\begin{array}{l}\text { Young's } \\
\text { Modulus }(\boldsymbol{E})\end{array}$ & $\begin{array}{l}\text { Poisson Ratio } \\
(\boldsymbol{v})\end{array}$ & $\begin{array}{l}\text { Yield } \\
\text { Strength }\left(\boldsymbol{S}_{\boldsymbol{y}}\right)\end{array}$ & $\begin{array}{l}\text { Critical } \\
\text { Interference } \\
\left(\omega_{\boldsymbol{c}} \boldsymbol{R}\right)\end{array}$ & $\gamma_{\boldsymbol{c}} / \boldsymbol{\omega}_{\boldsymbol{c}}(\boldsymbol{R}=\mathbf{1})$ \\
\hline Aluminum & $70 \mathrm{GPa}$ & 0.33 & $0.310 \mathrm{GPa}$ & $1.03 \cdot 10^{-4}$ & $1.69 \cdot 10^{-3}$ \\
\hline Steel & $200 \mathrm{GPa}$ & 0.32 & $1.619 \mathrm{GPa}$ & $3.50 \cdot 10^{-4}$ & $3.12 \cdot 10^{-3}$ \\
\hline
\end{tabular}

Table 2: The location and value of the maximum von Mises residual stress for various normalized interference depths.

\begin{tabular}{|l|l|l|l|}
\hline $\begin{array}{l}\text { Normalized } \\
\text { Interference } \\
\text { Depth }\left(\omega^{*}\right)\end{array}$ & $\begin{array}{l}\text { Maximum } \\
\text { Unloaded von } \\
\text { Mises Stress } \\
\left(\sigma_{v m} / \boldsymbol{S}_{\boldsymbol{y}}\right)\end{array}$ & ${\boldsymbol{r} / \boldsymbol{a}_{\boldsymbol{c}}}$ & $\boldsymbol{( R - y ) / \omega _ { c }}$ \\
\hline 1.43 & 0.057 & 0.00 & 34.18 \\
\hline 2.14 & 0.217 & 0.00 & 43.44 \\
\hline 3.57 & 0.344 & 0.00 & 61.64 \\
\hline 3.92 & 0.371 & 1.90 & 1.81 \\
\hline 4.29 & 0.408 & 2.02 & 2.07 \\
\hline 5.00 & 0.511 & 2.29 & 2.61 \\
\hline 5.72 & 0.615 & 2.46 & 3.03 \\
\hline 8.57 & 0.693 & 3.24 & 5.26 \\
\hline 10.00 & 0.754 & 3.73 & 6.97 \\
\hline 15.00 & 0.883 & 4.70 & 11.05 \\
\hline 17.50 & 0.952 & 5.11 & 13.11 \\
\hline 20.00 & 0.986 & 5.59 & 15.65 \\
\hline 25.00 & 0.994 & 6.38 & 20.46 \\
\hline 30.00 & 1.000 & 7.25 & 33.57 \\
\hline
\end{tabular}




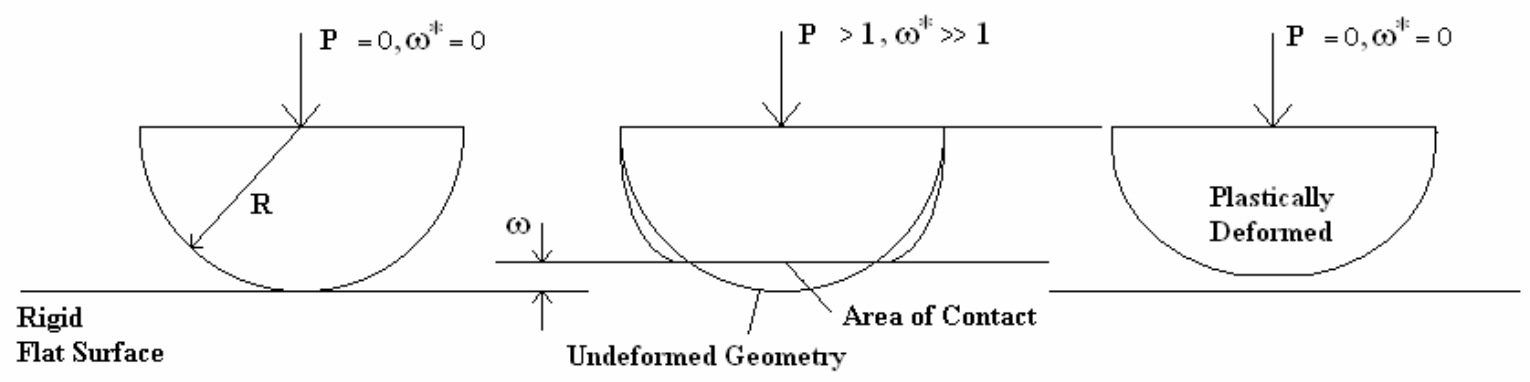

(a)

(b)

(c)

Figure 1: Diagram of loaded (b) and unloaded (c) contact of deforming elasto-plastic hemispheres and a rigid flat. 


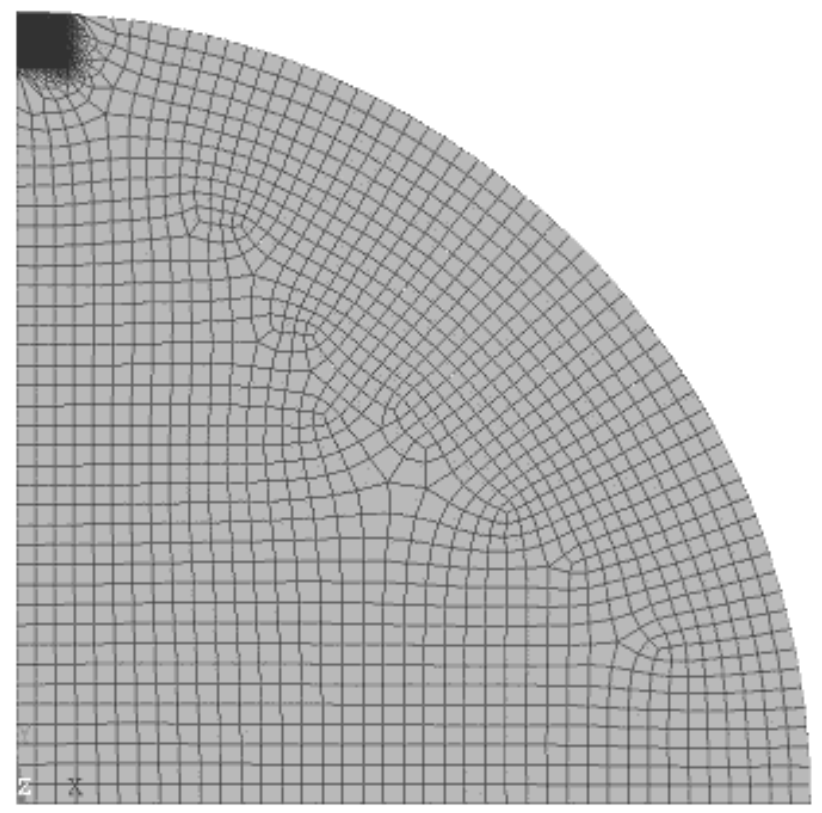

Figure 2: Example of used FEM mesh. 


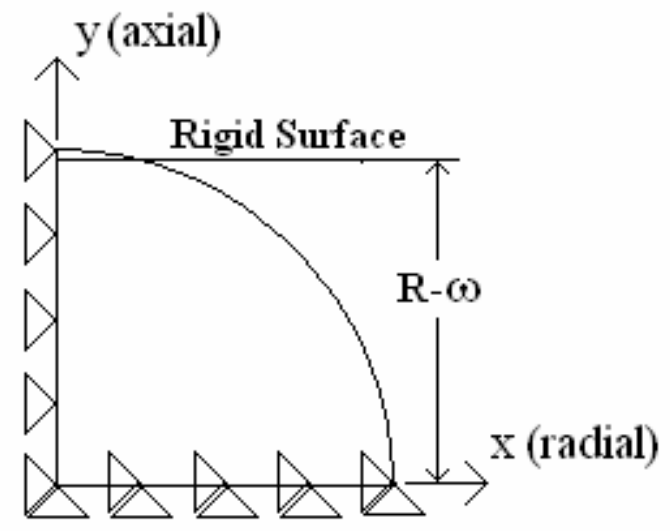

Figure 3: Schematic of the coordinate system and boundary conditions used in the FEM. 


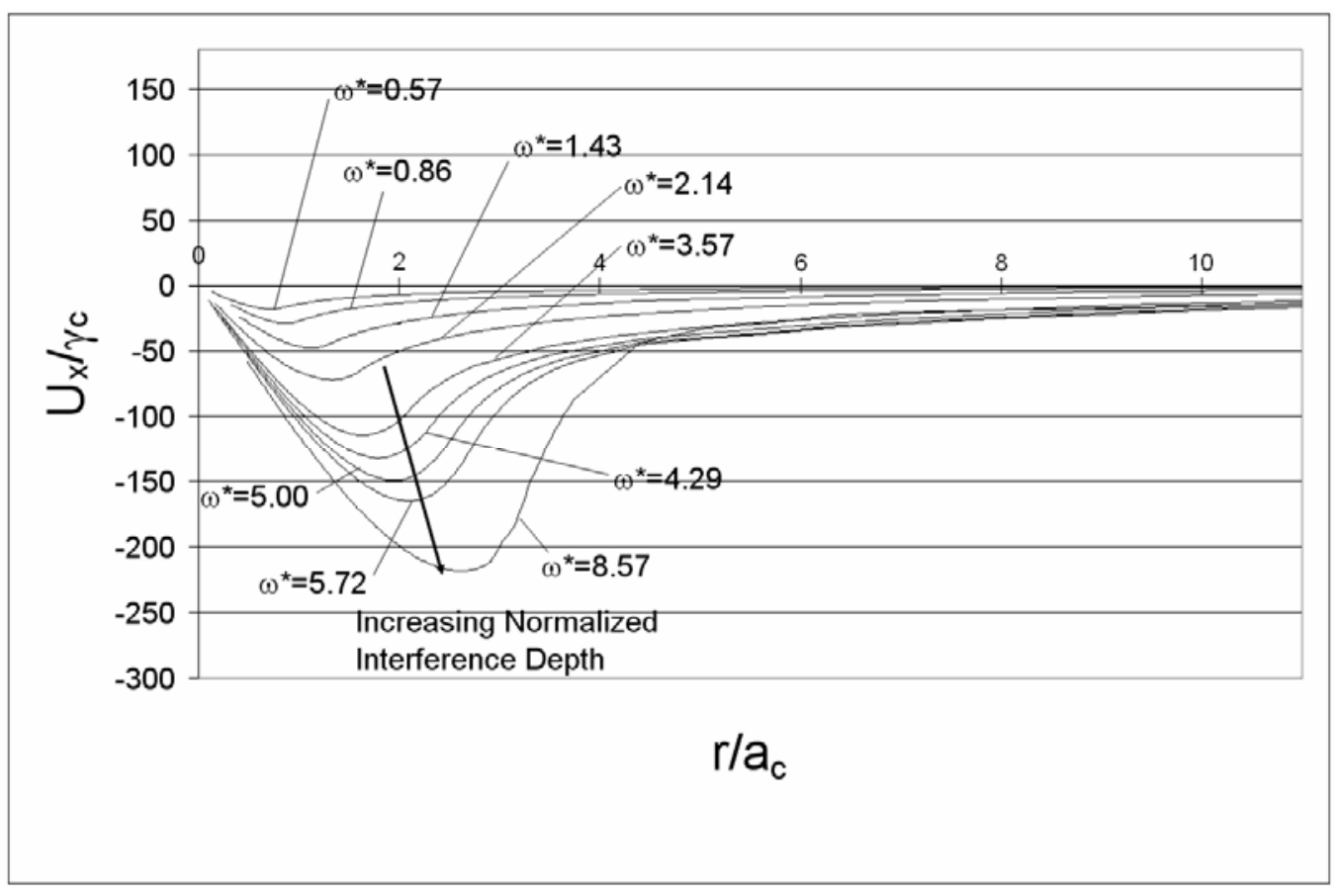

(a) small normalized loaded interferences, $\omega^{*}$

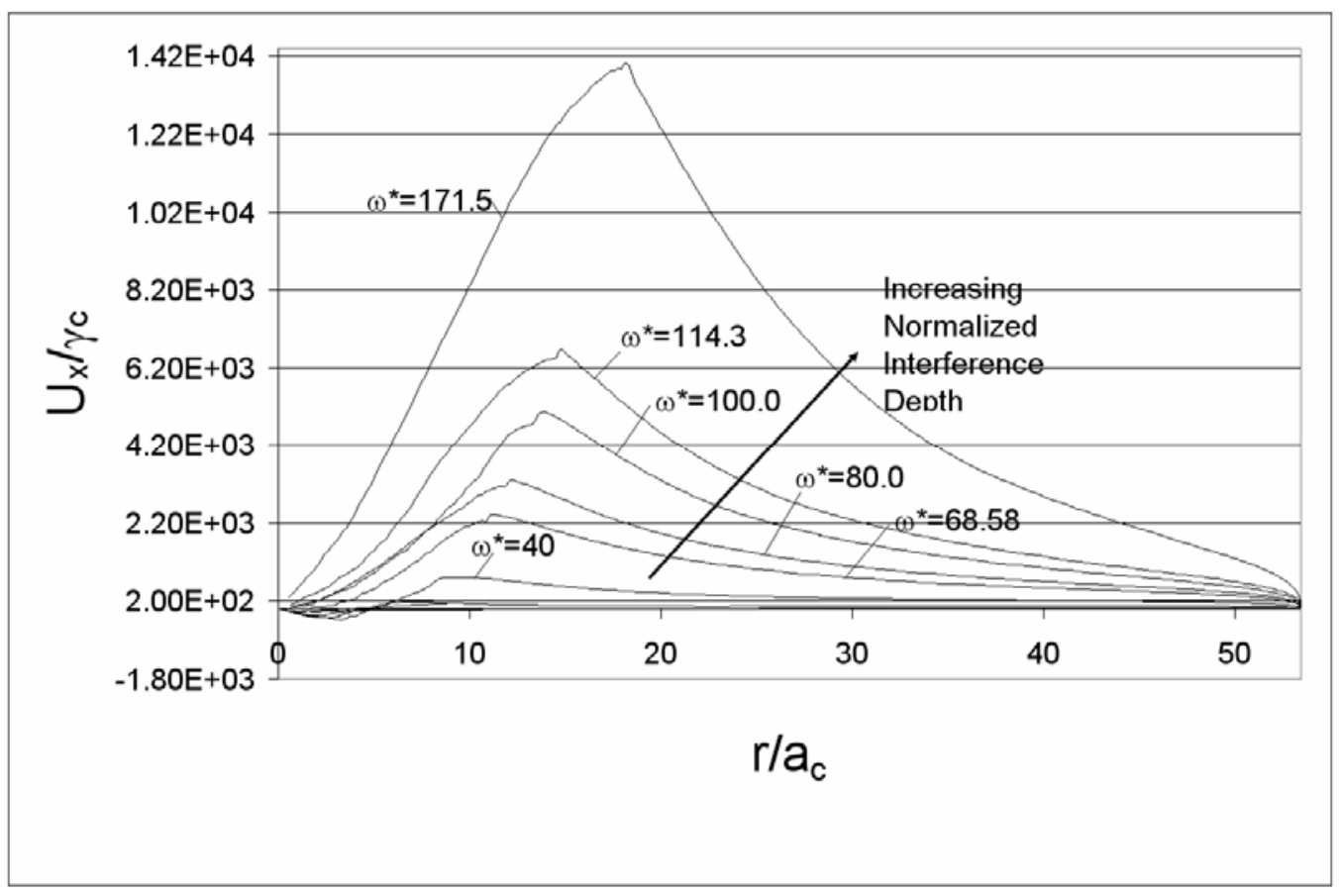

(b) large normalized loaded interferences, $\omega^{*}$

Figure 4: The normalized radial surface displacement versus the normalized radial distance in the loaded condition for (a) small, and (b) large normalized interference depths. 


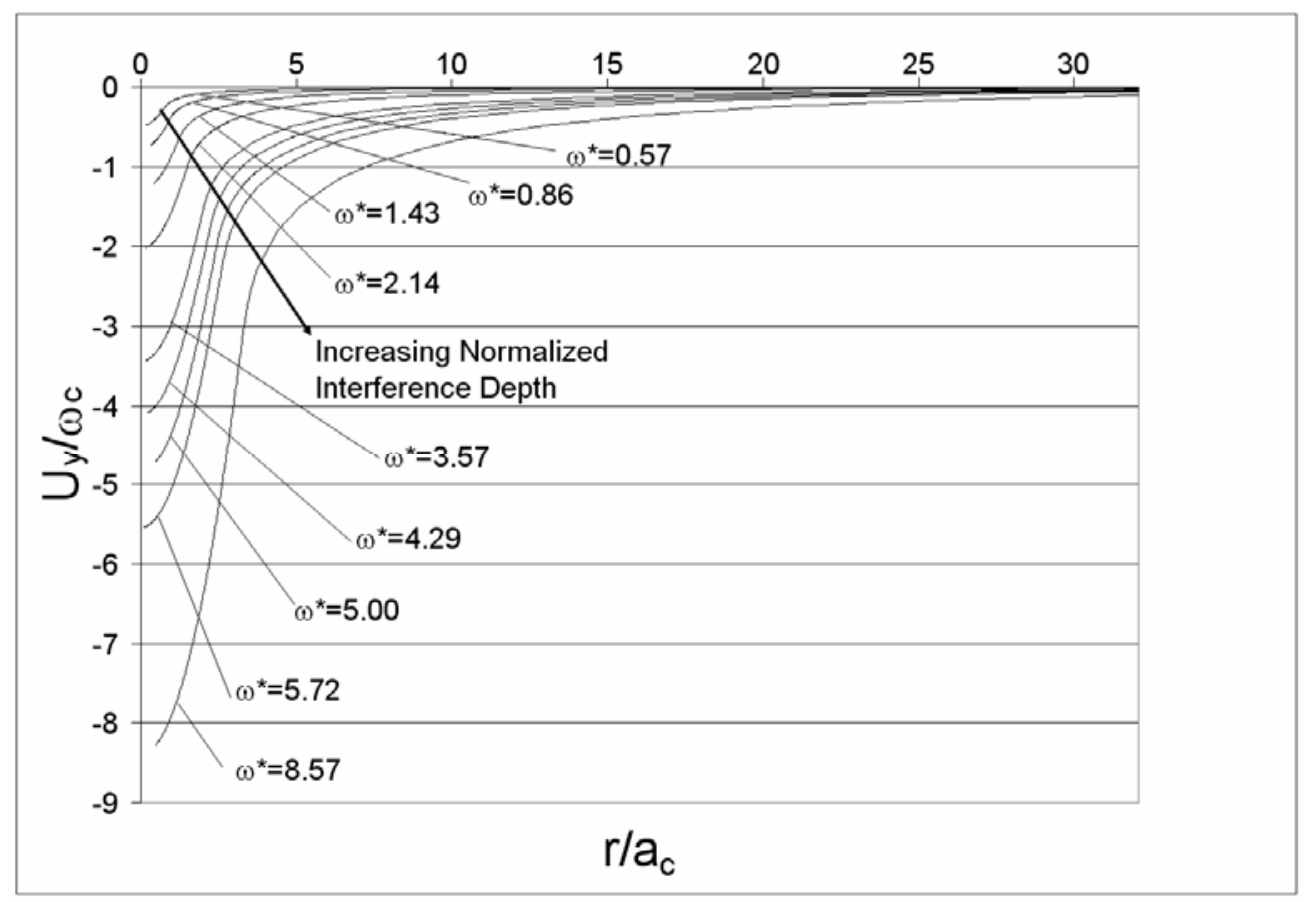

(a) small normalized loaded interferences, $\omega^{*}$

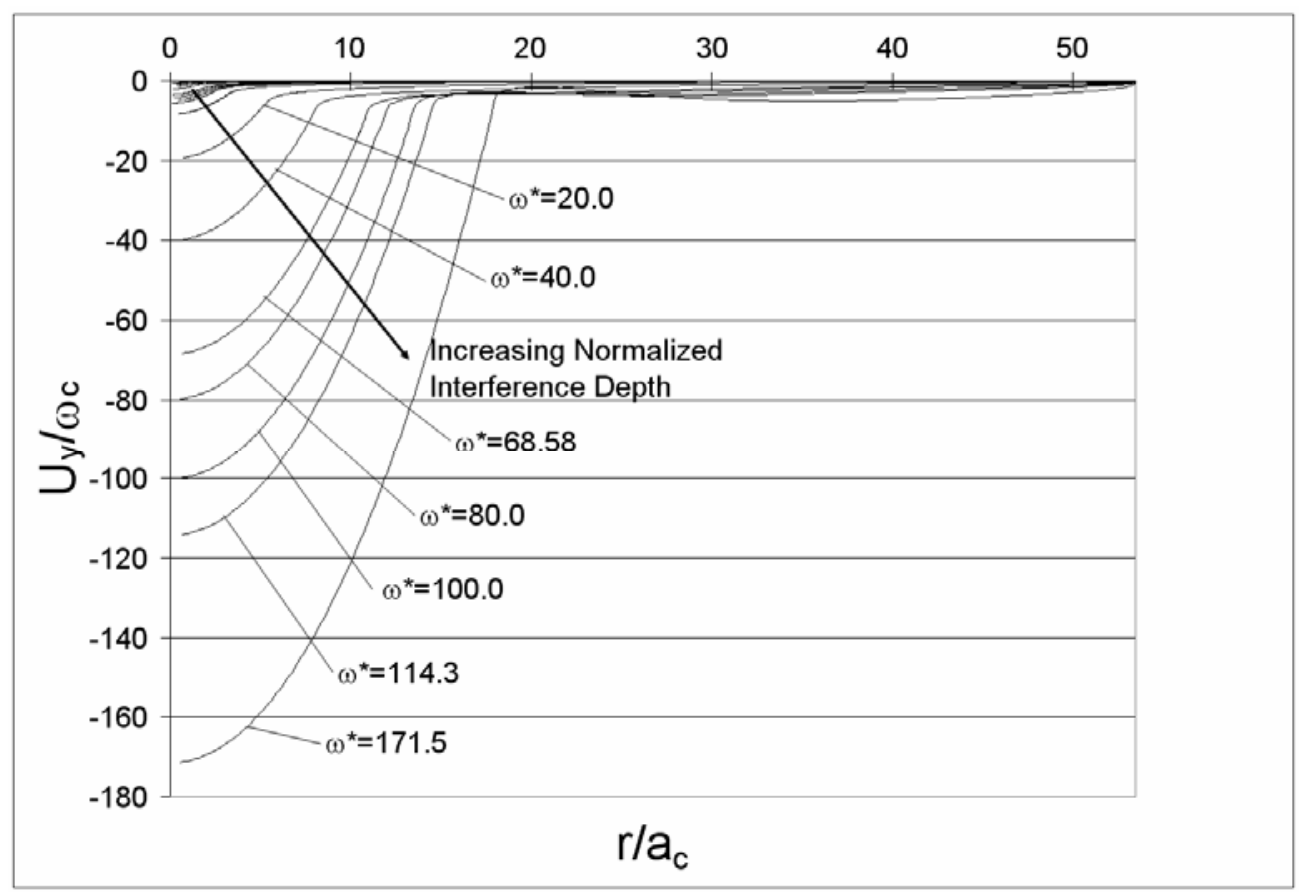

(b) large normalized loaded interferences, $\omega^{*}$

Figure 5: The normalized axial displacement versus the normalized radial distance in the loaded condition for (a) small, and (b) large normalized interference depths. 


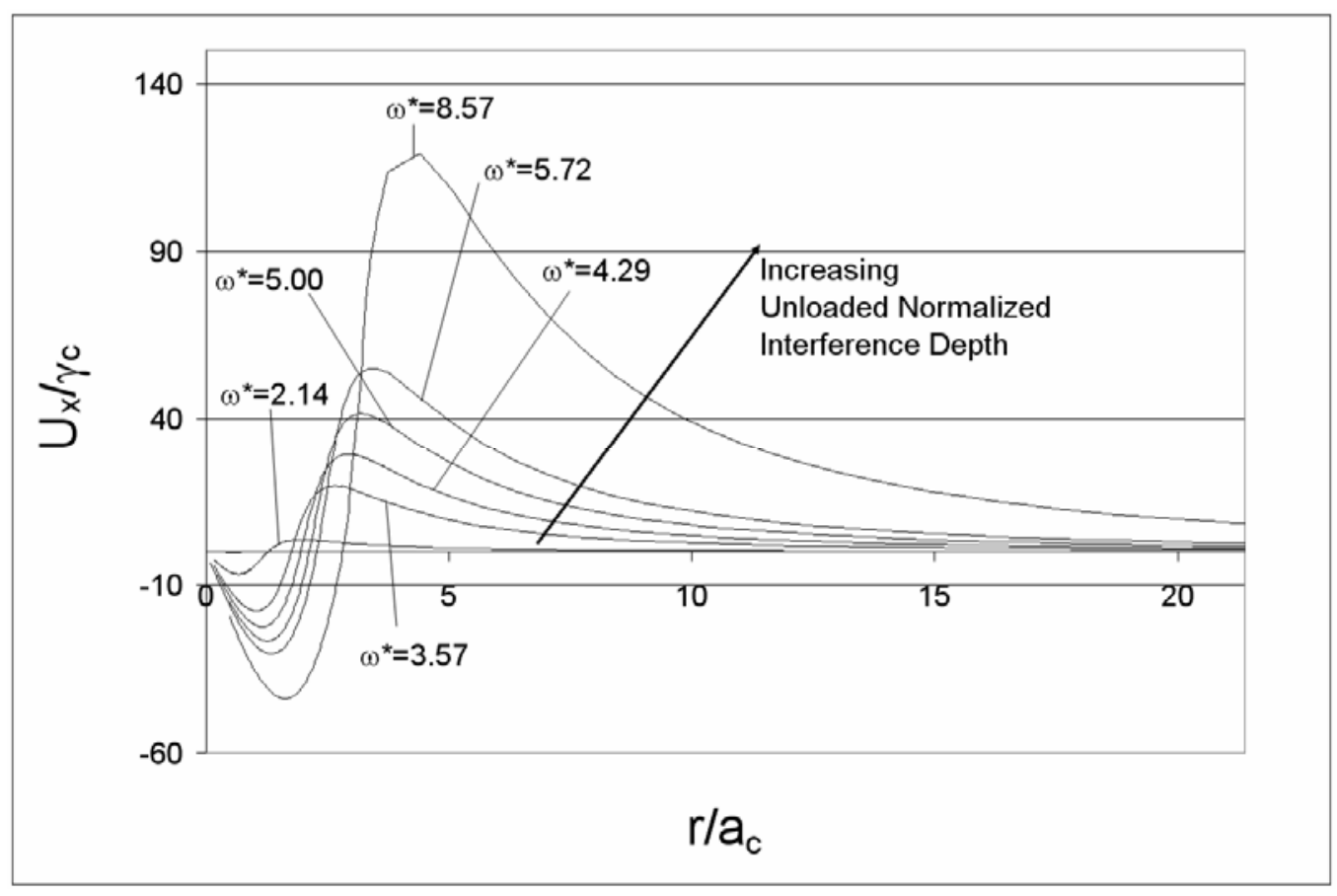

(a) small normalized unloaded interferences, $\omega^{*}$

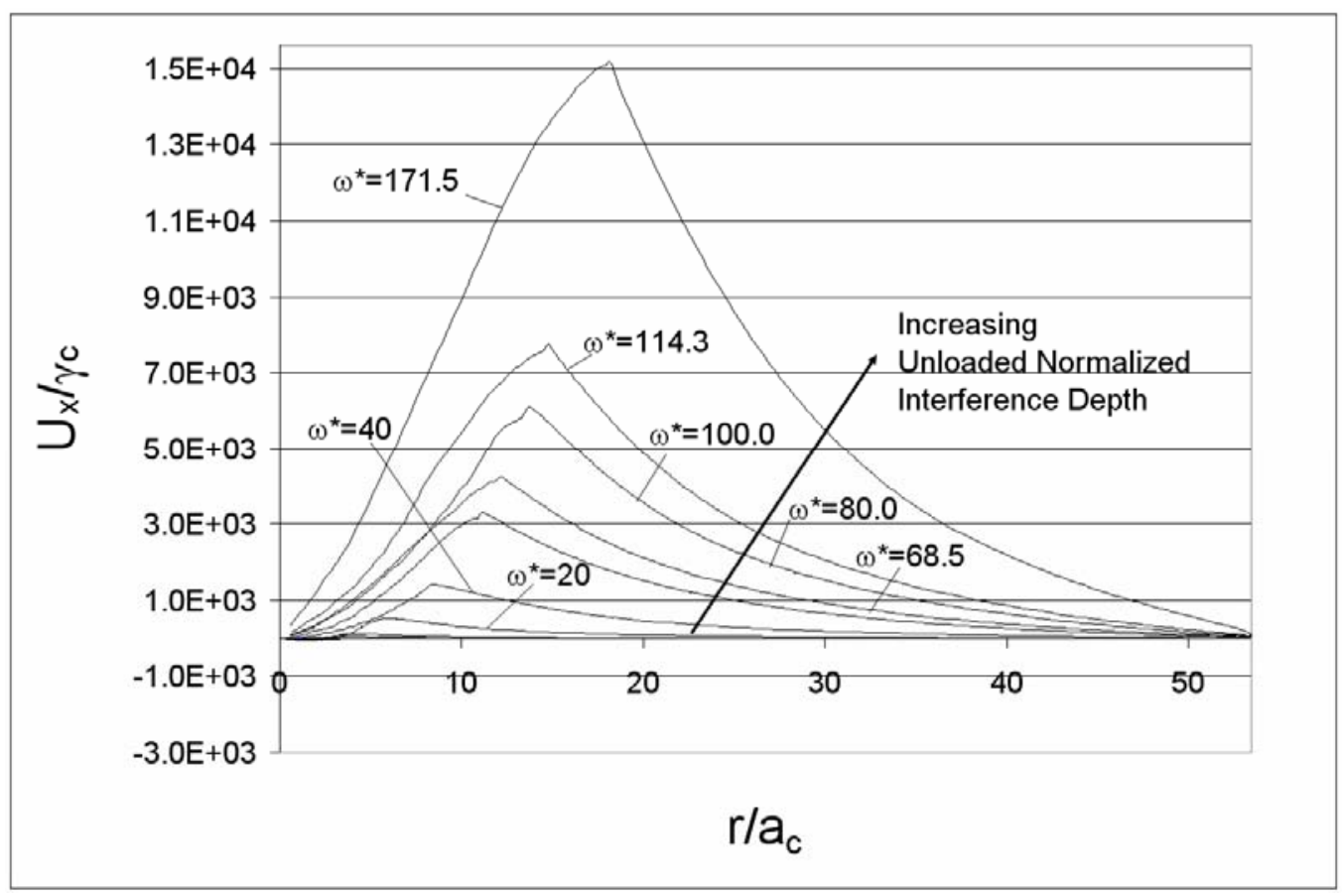

(b) large normalized unloaded interferences, $\omega^{*}$

Figure 6: The normalized radial residual displacement versus the normalized radial distance of the hemisphere unloaded from (a) small, and (b) large normalized interference depths. 


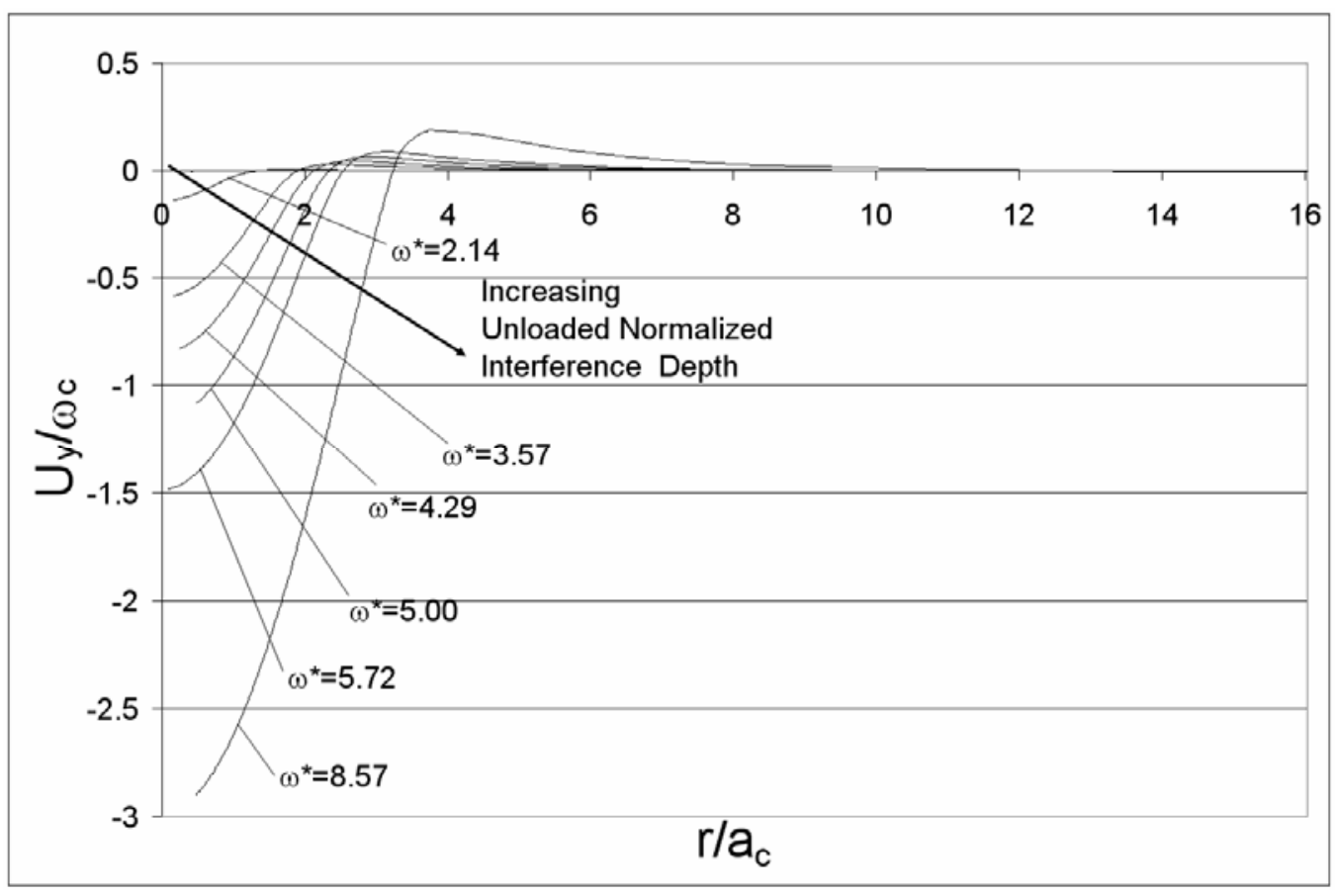

(a) small normalized unloaded interferences, $\omega^{*}$

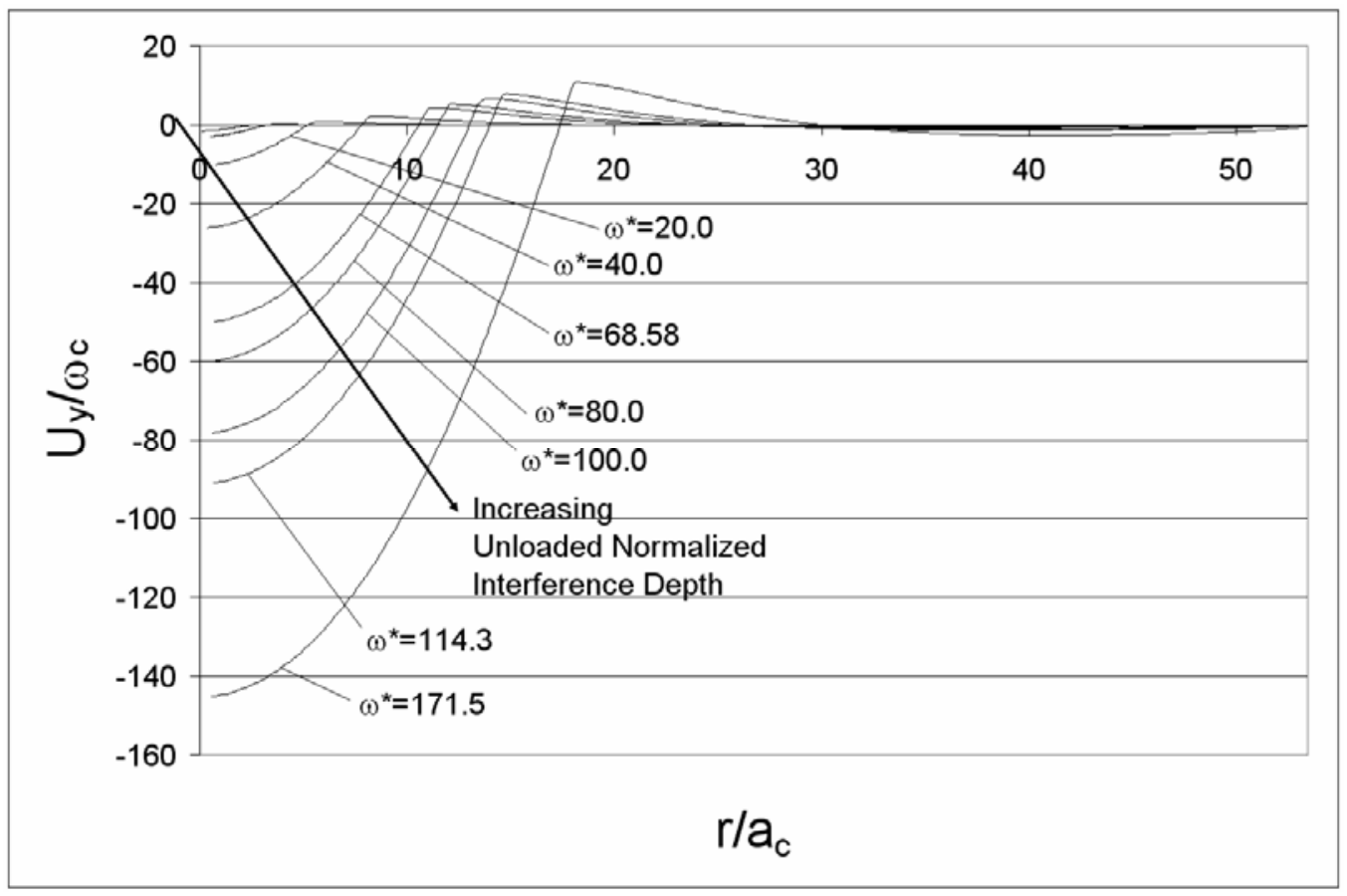

(b) large normalized unloaded interferences, $\omega^{*}$

Figure 7: The normalized axial displacement versus the normalized radial distance of the hemisphere unloaded from (a) small, and (b) large normalized interference depths. 


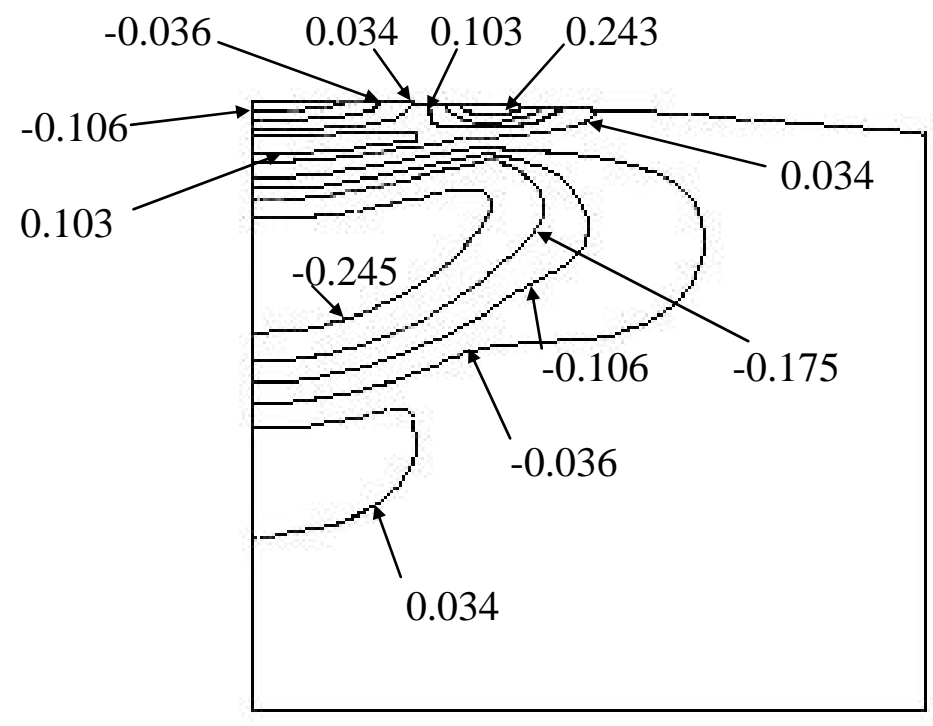

(a) radial stress, $\sigma_{x} / S_{y}$

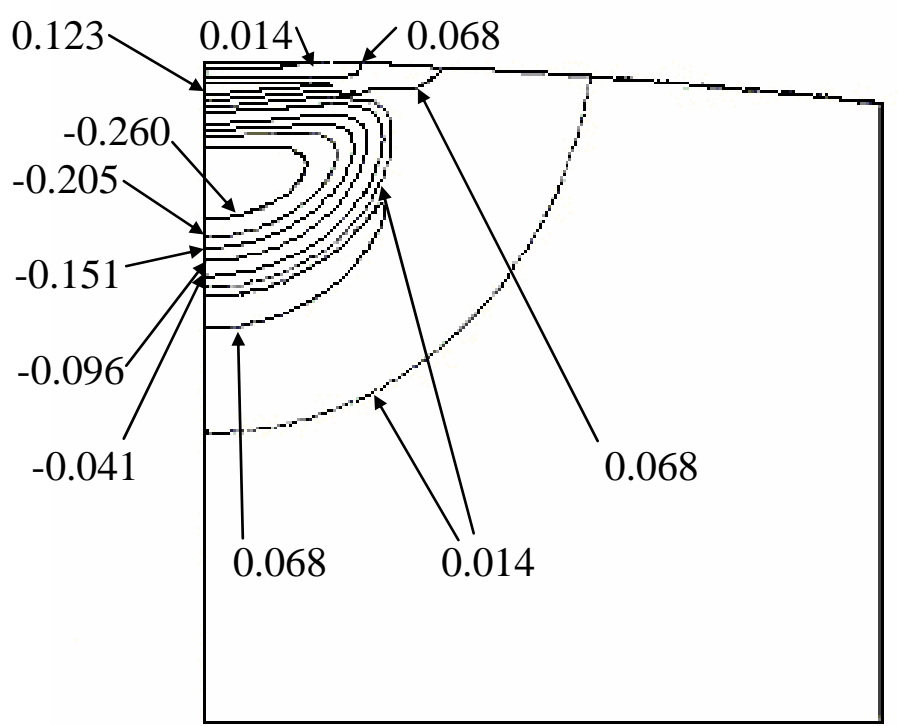

(c) hoop stress, $\sigma_{z} / S_{y}$

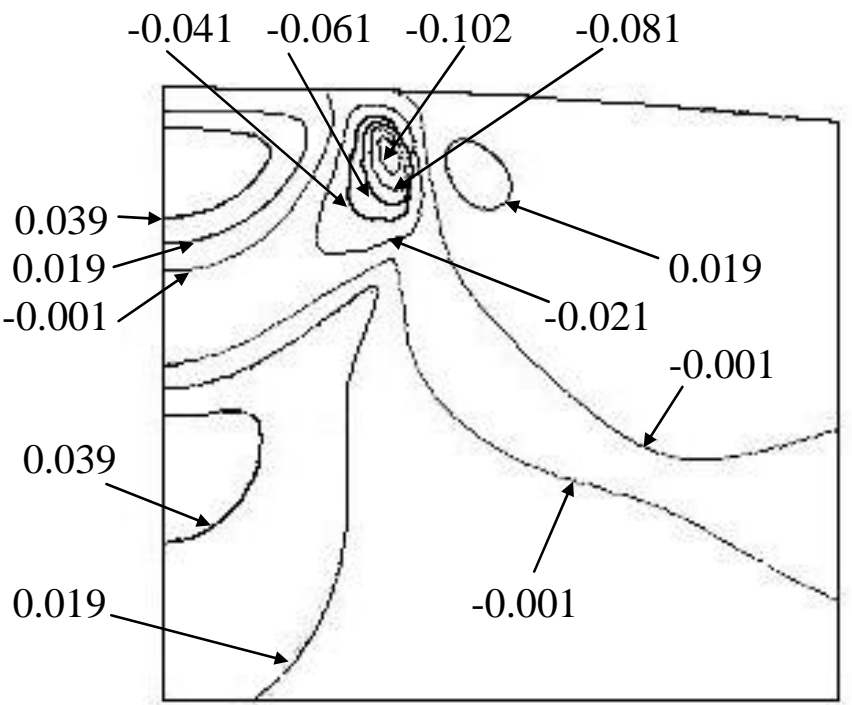

(b) axial stress, $\sigma_{y} / S_{y}$

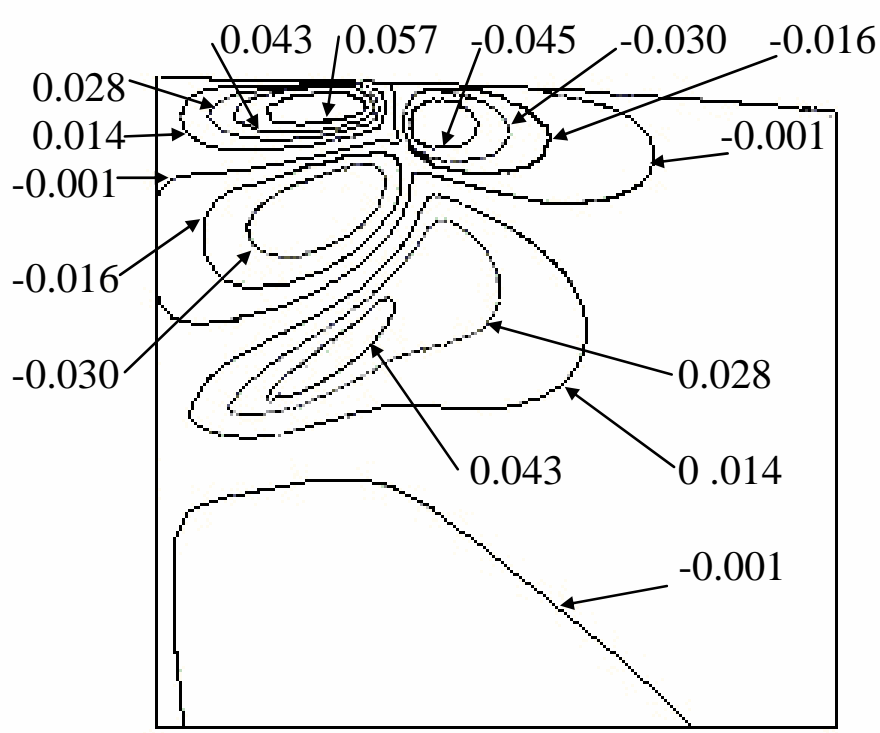

(d) shear stress, $\tau_{x y} / S_{y}$

Figure 8: Contour plots of the complete stress tensor for a hemispherical contact unloaded from $\omega^{*}=3.92$. 


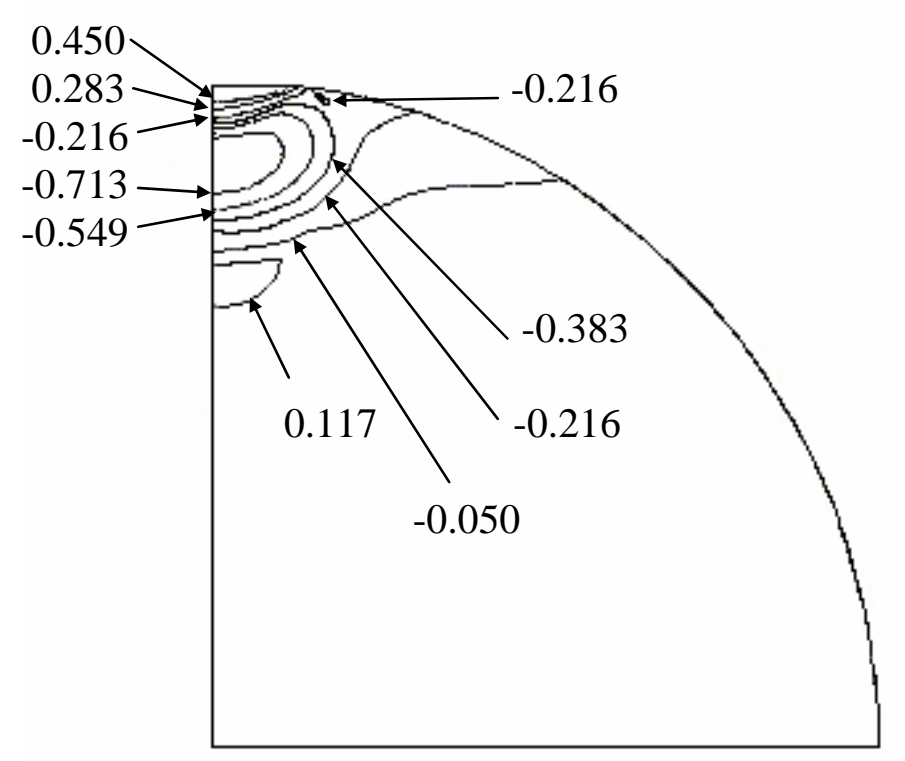

(a) radial stress, $\sigma_{x} / S_{y}$

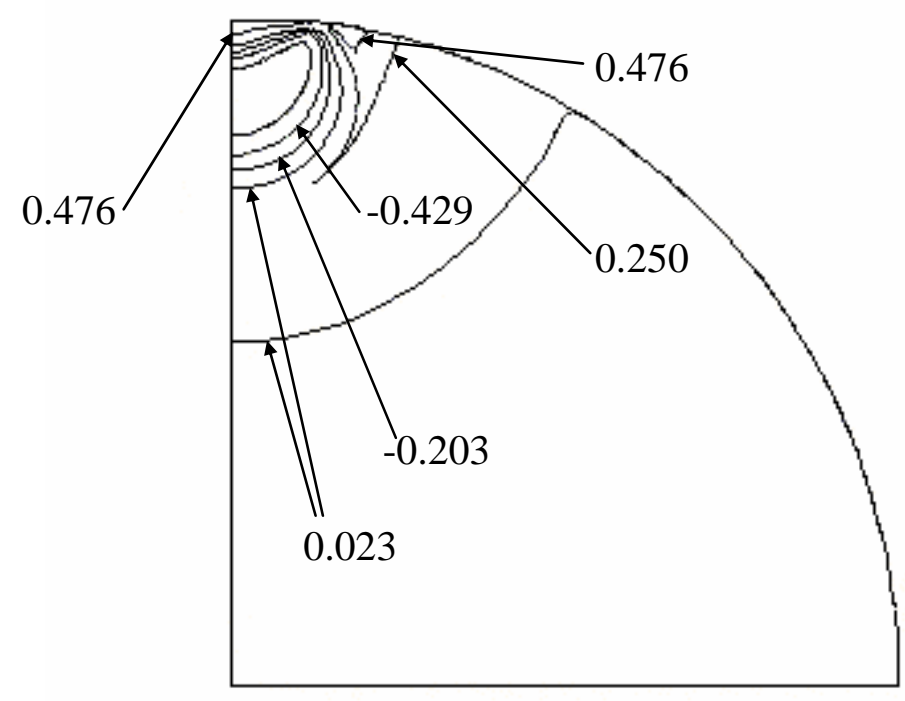

(c) hoop stress, $\sigma_{z} / S_{y}$

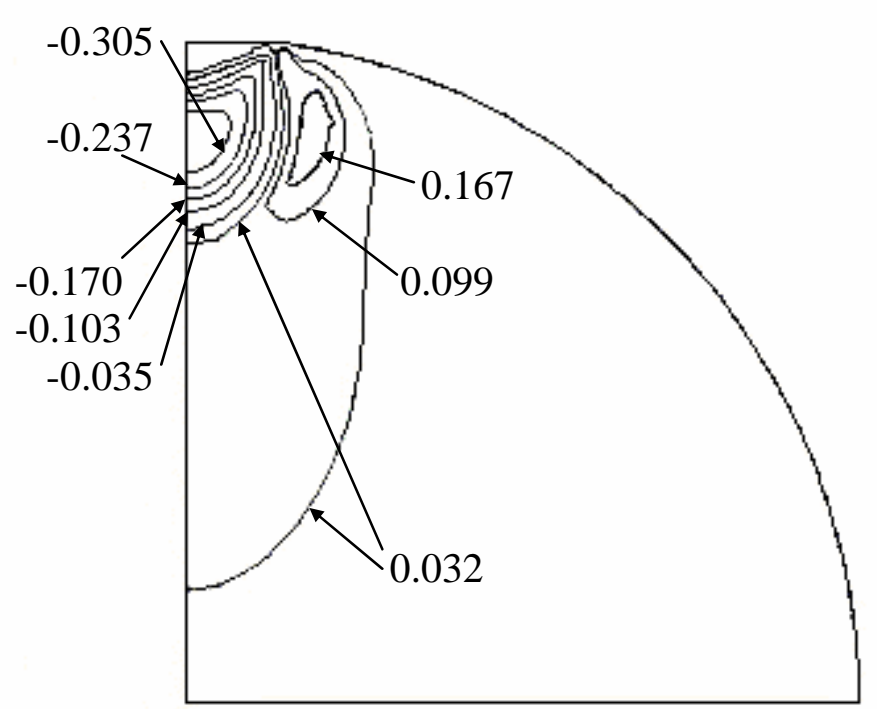

(b) axial stress, $\sigma_{y} / S_{y}$

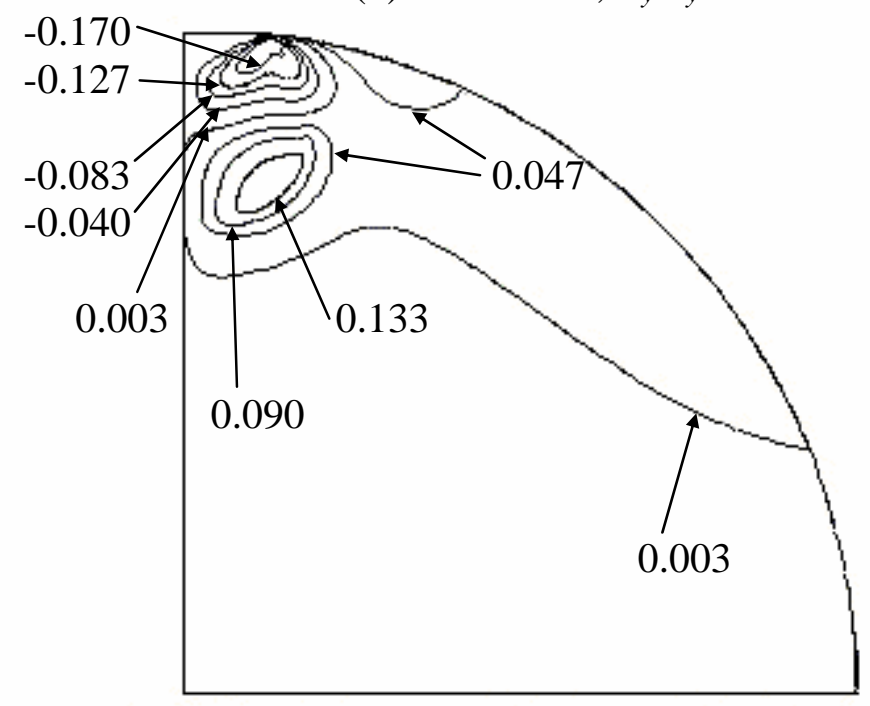

(d) shear stress, $\tau_{x y} / S_{y}$

Figure 9: Contour plots of the complete stress tensor for a hemispherical contact unloaded from $\omega^{*}=35.0$. 


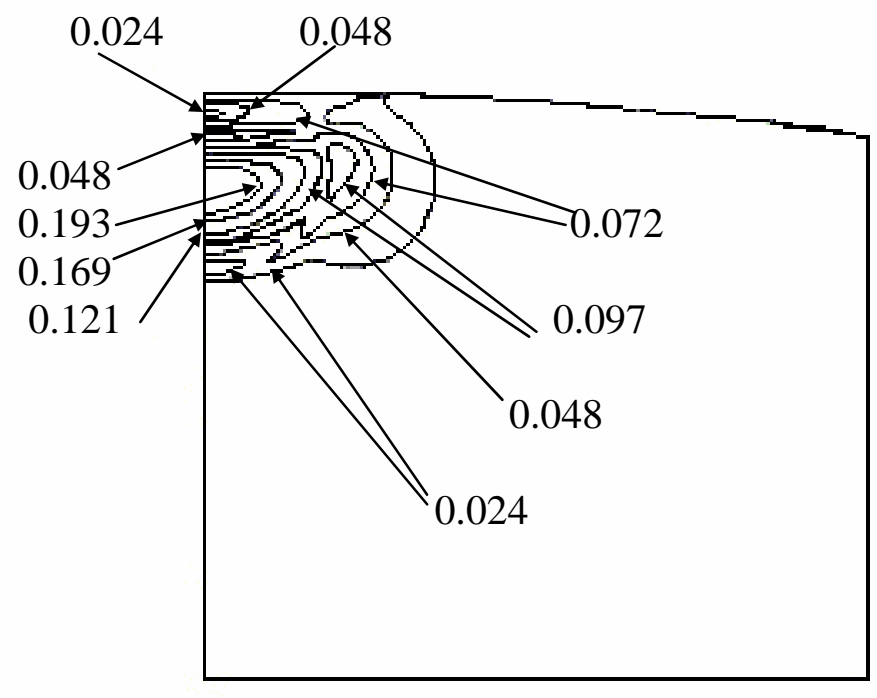

(a) $\omega^{*}=2.14$

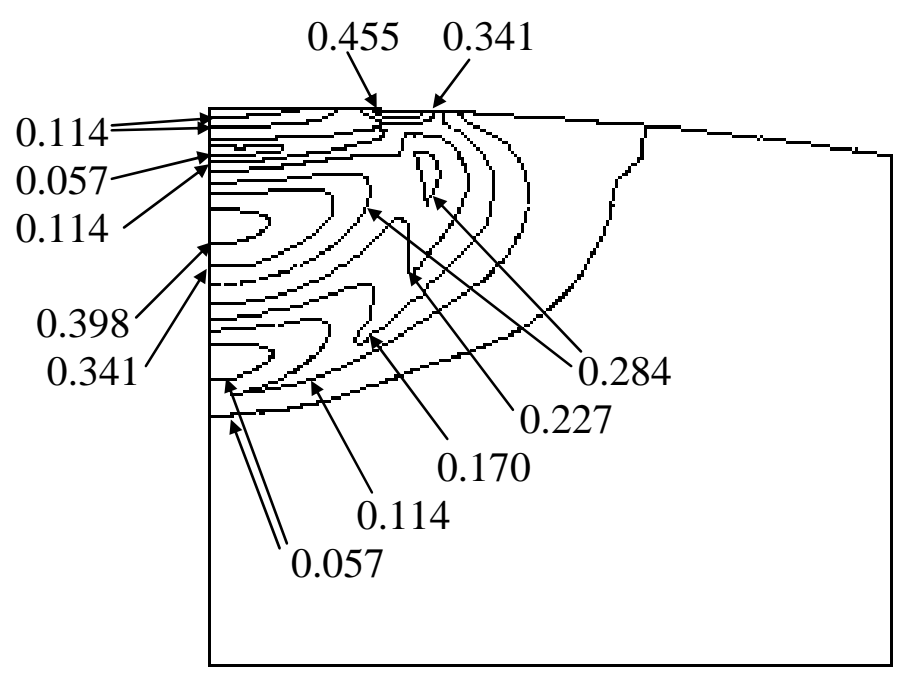

(c) $\omega^{*}=5.71$

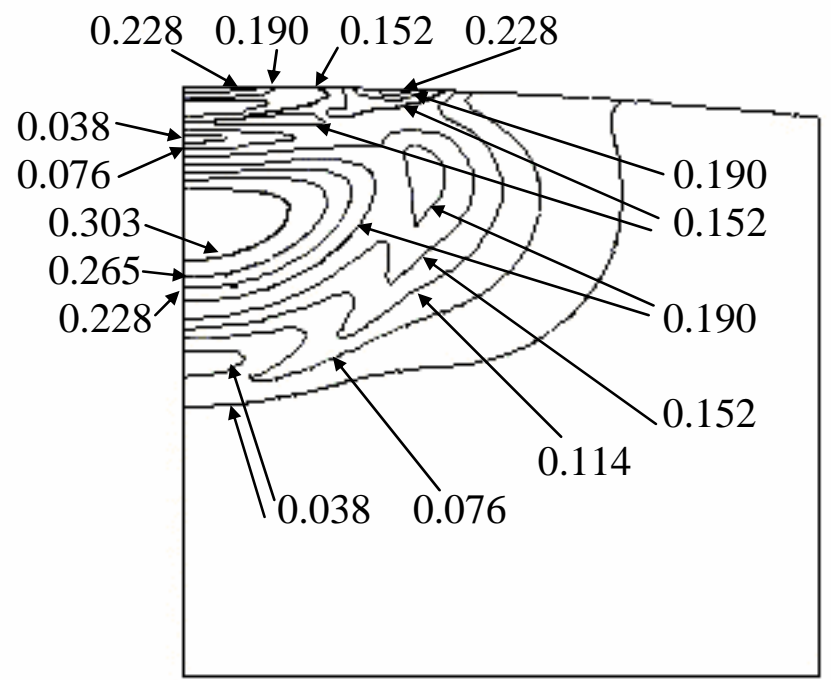

(b) $\omega^{*}=3.92$

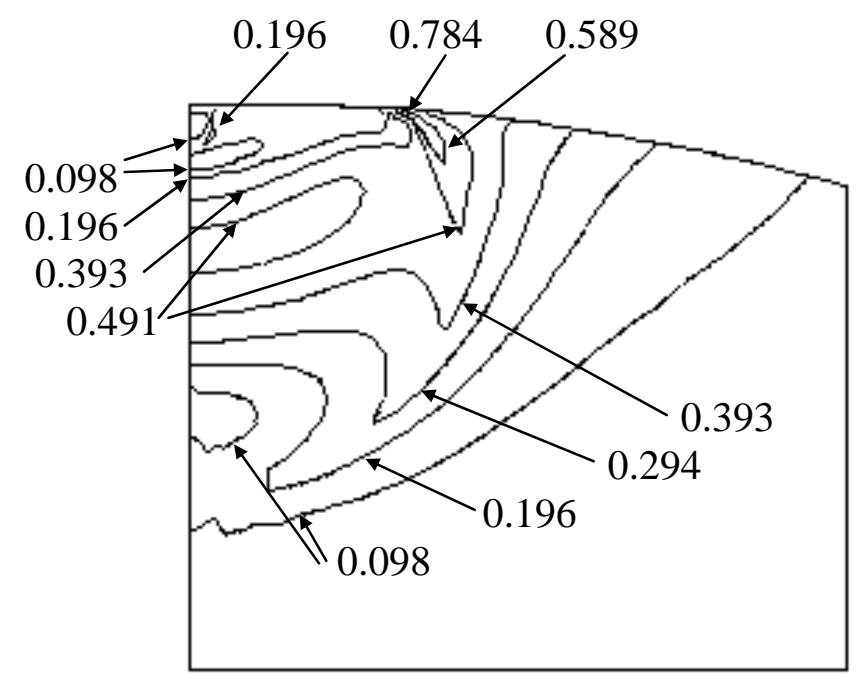

(d) $\omega^{*}=15.00$

Figure 10: Contour plot of the normalized elastic residual von Mises stress $\left(\sigma_{v m} / S_{y}\right)$ at various unloaded normalized interferences. 


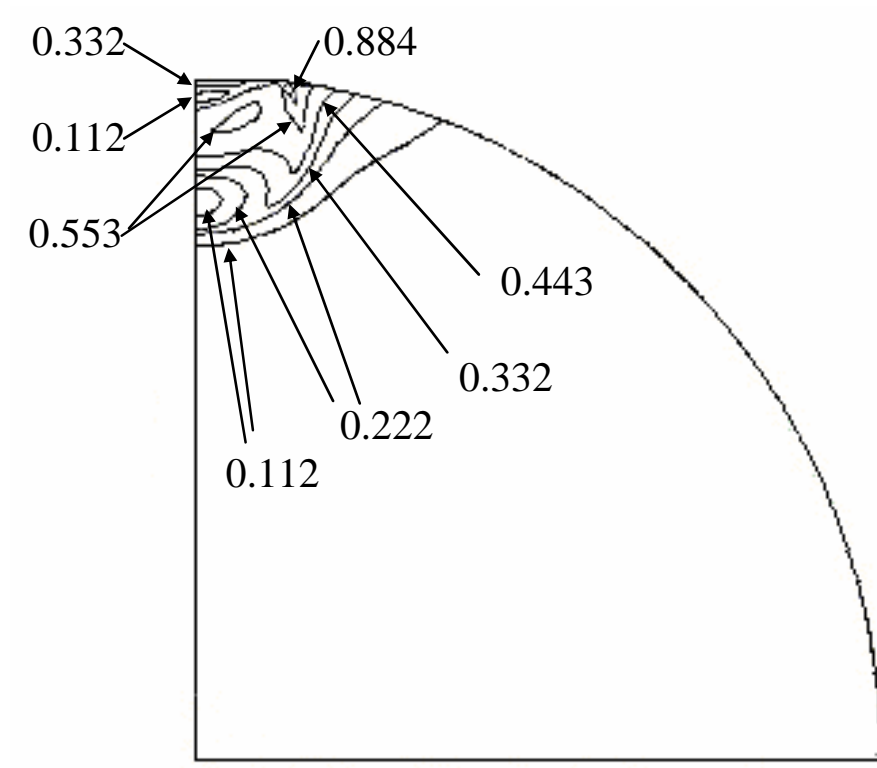

(a) $\omega^{*}=25.0$

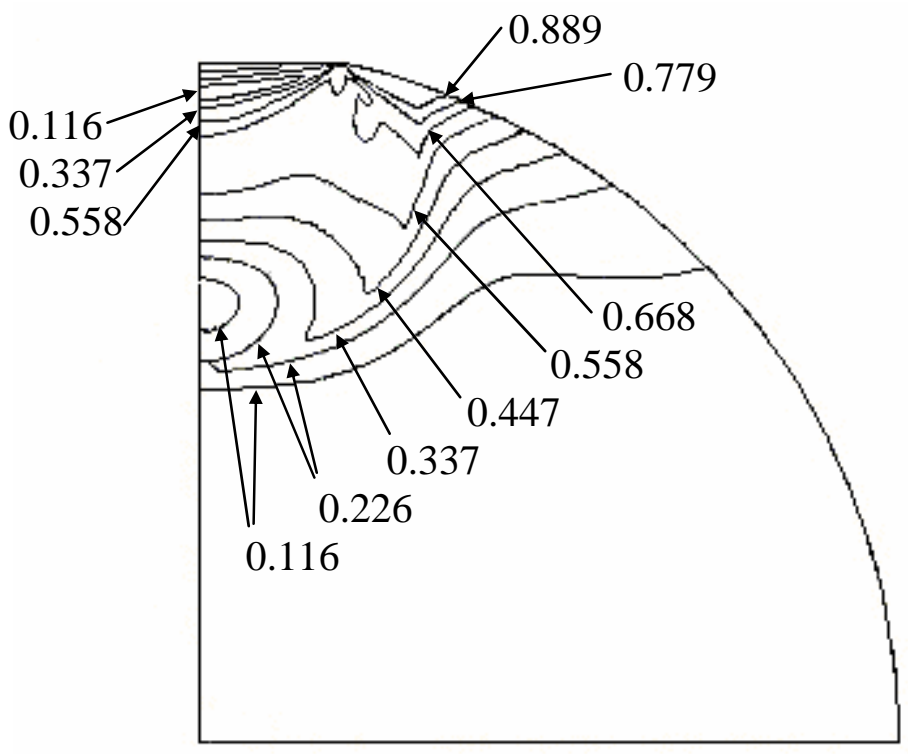

(c) $\omega^{*}=68.6$

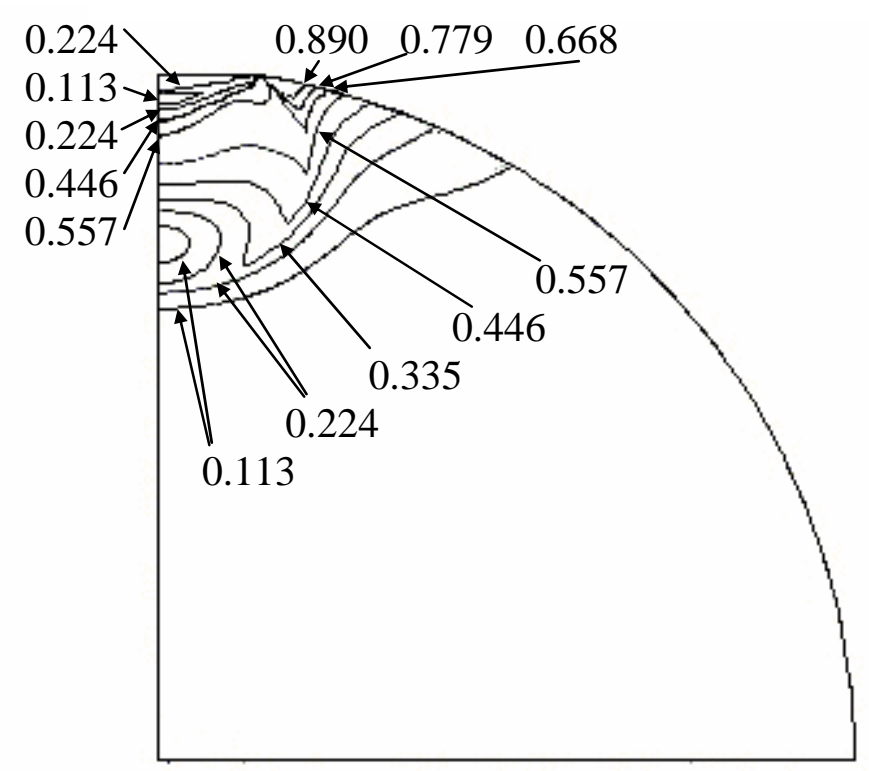

(b) $\omega^{*}=40.0$

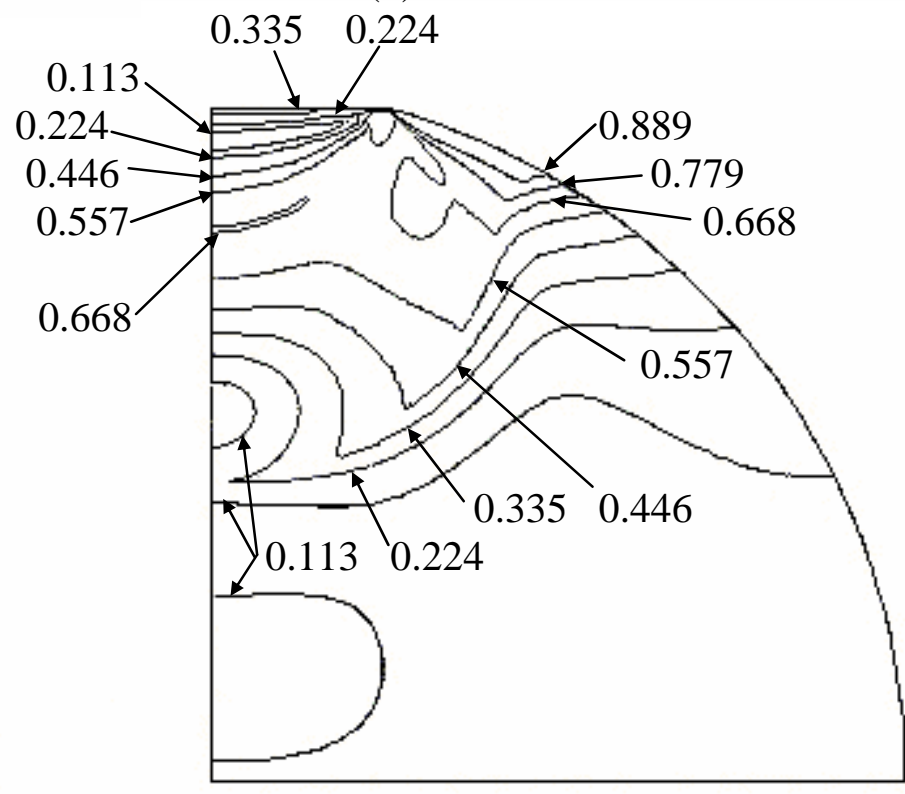

(d) $\omega^{*}=100.0$

Figure 11: Contour plot of the normalized residual von Mises stress $\left(\sigma_{v m} / S_{y}\right)$ at various unloaded normalized interferences at the onset and formation of plastic residual stresses. 


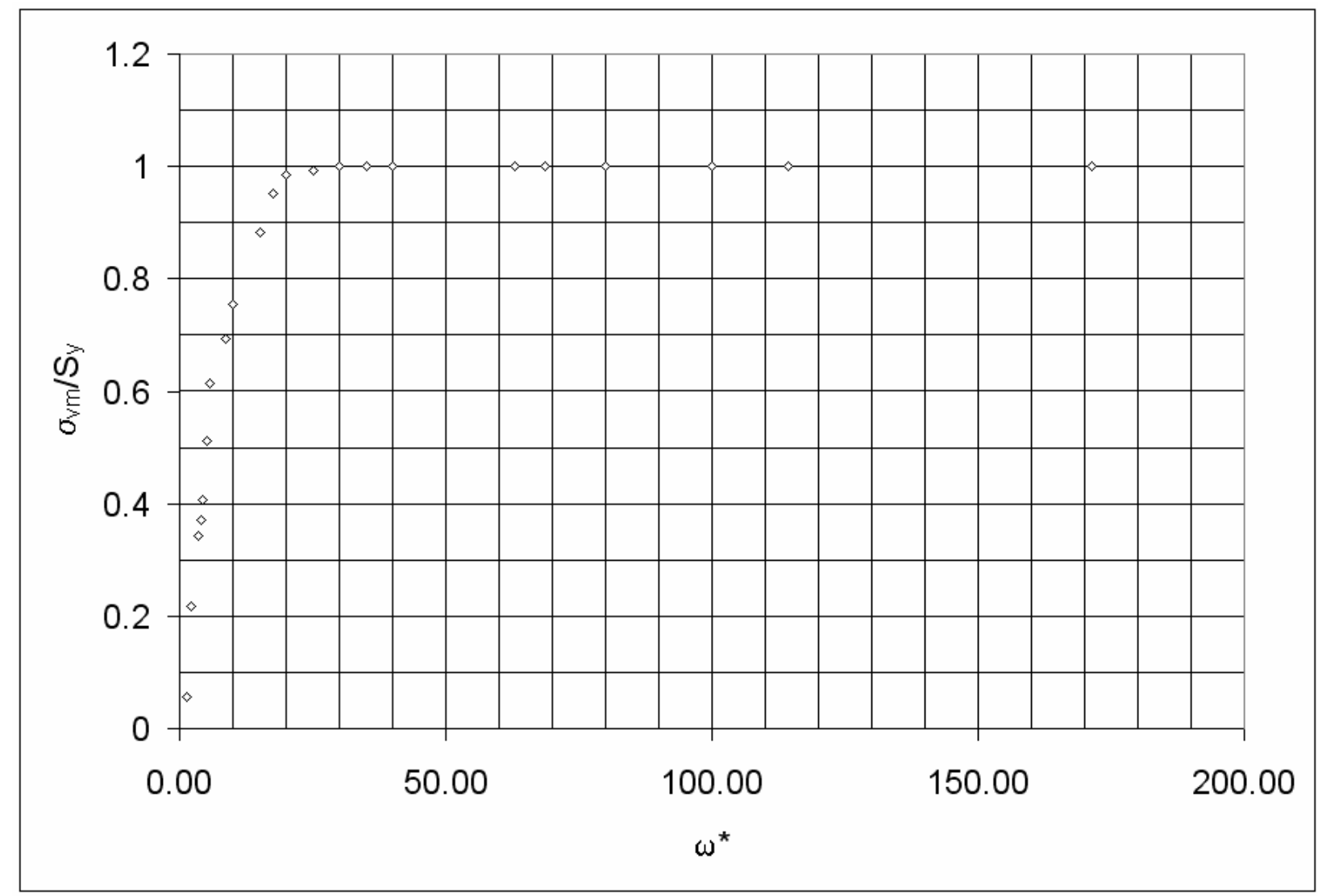

Figure 12: The normalized maximum von Mises residual stress of the unloaded hemisphere as a function of the unloaded $\omega^{*}$. 


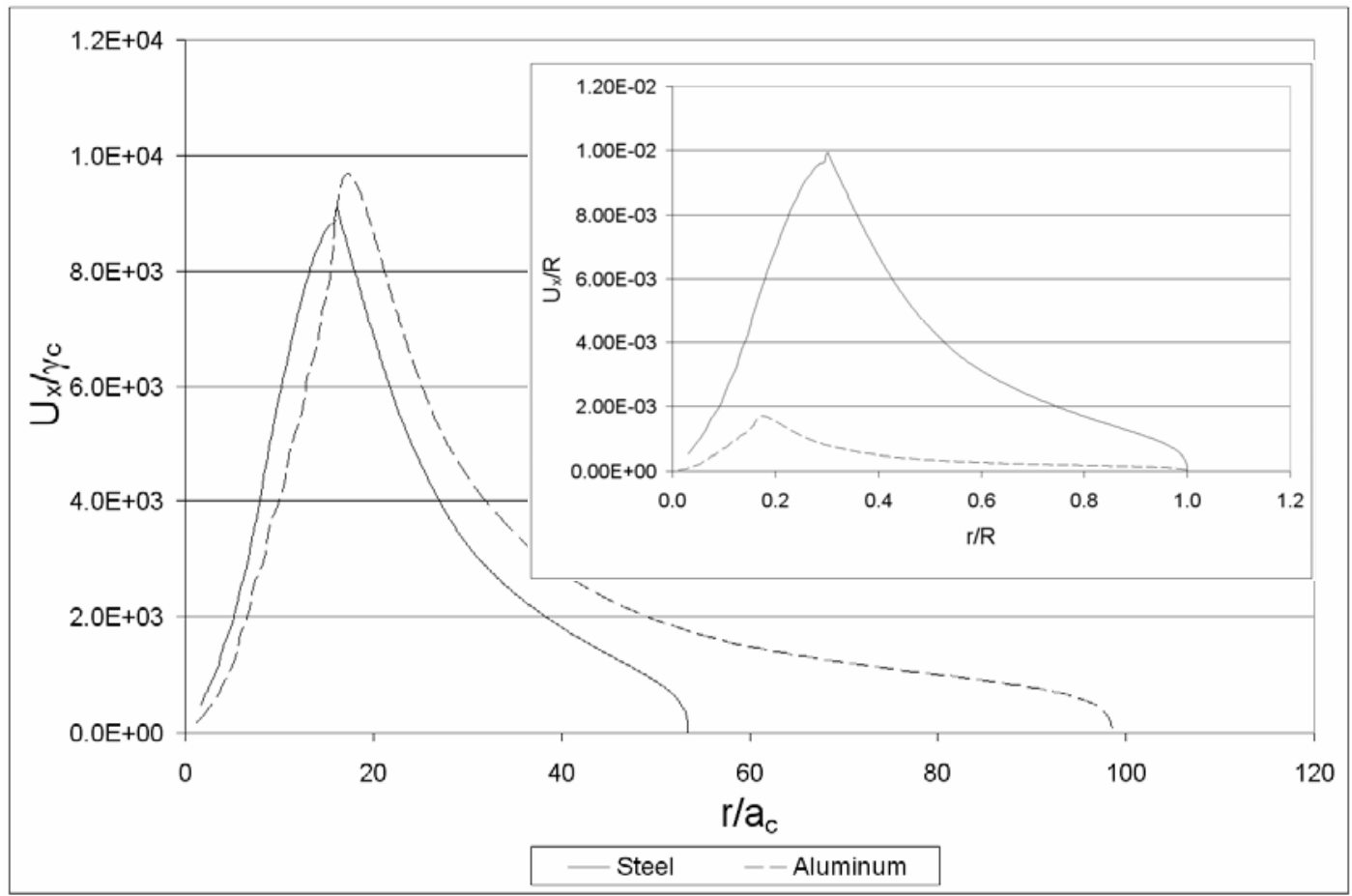

(a) Loaded Radial Displacement

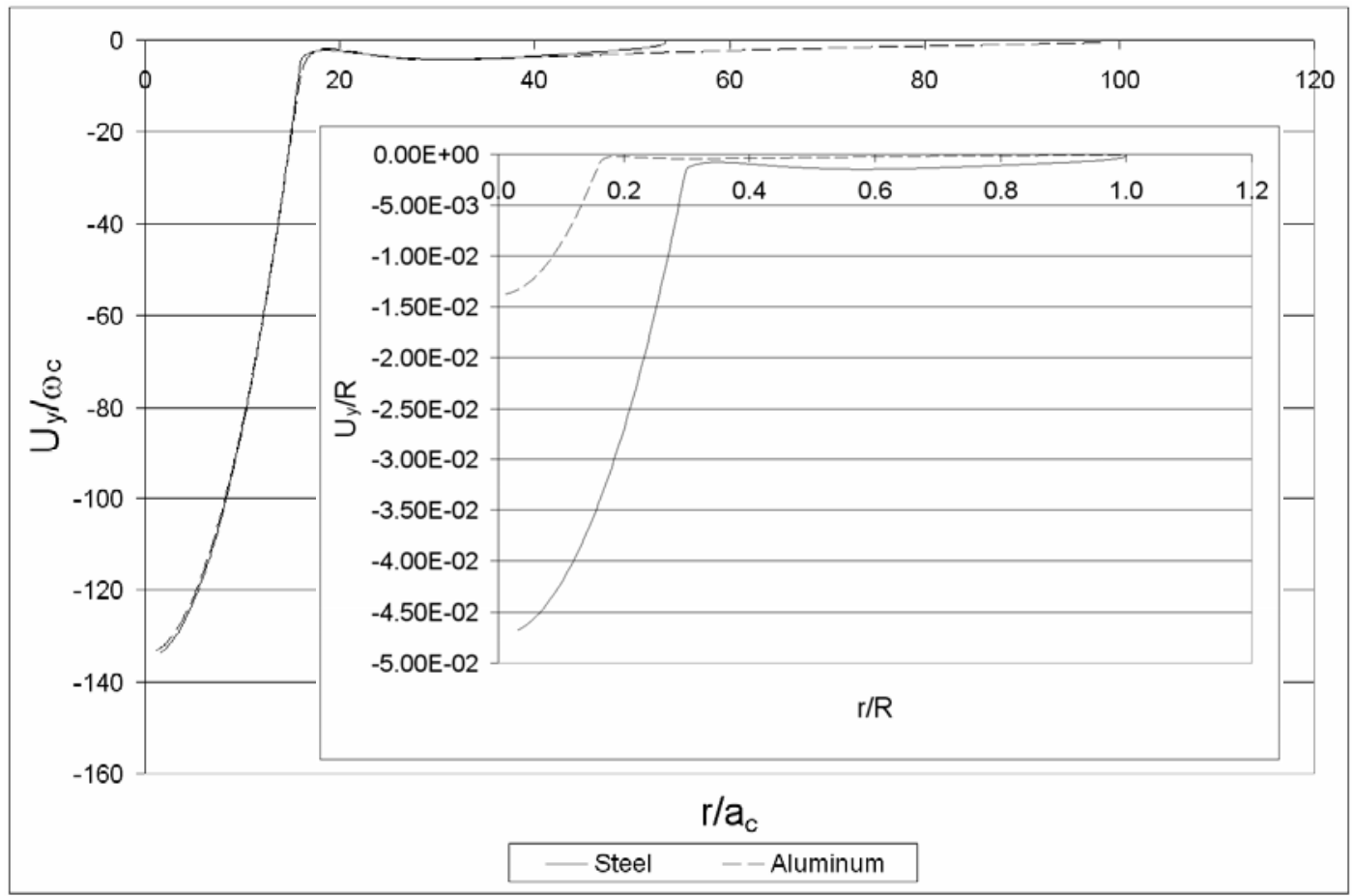

(b) Loaded Axial Displacement

Figure 13: The normalized surface displacement of aluminum and steel hemispheres loaded to $\omega^{*}=135$ versus the normalized radial location. 


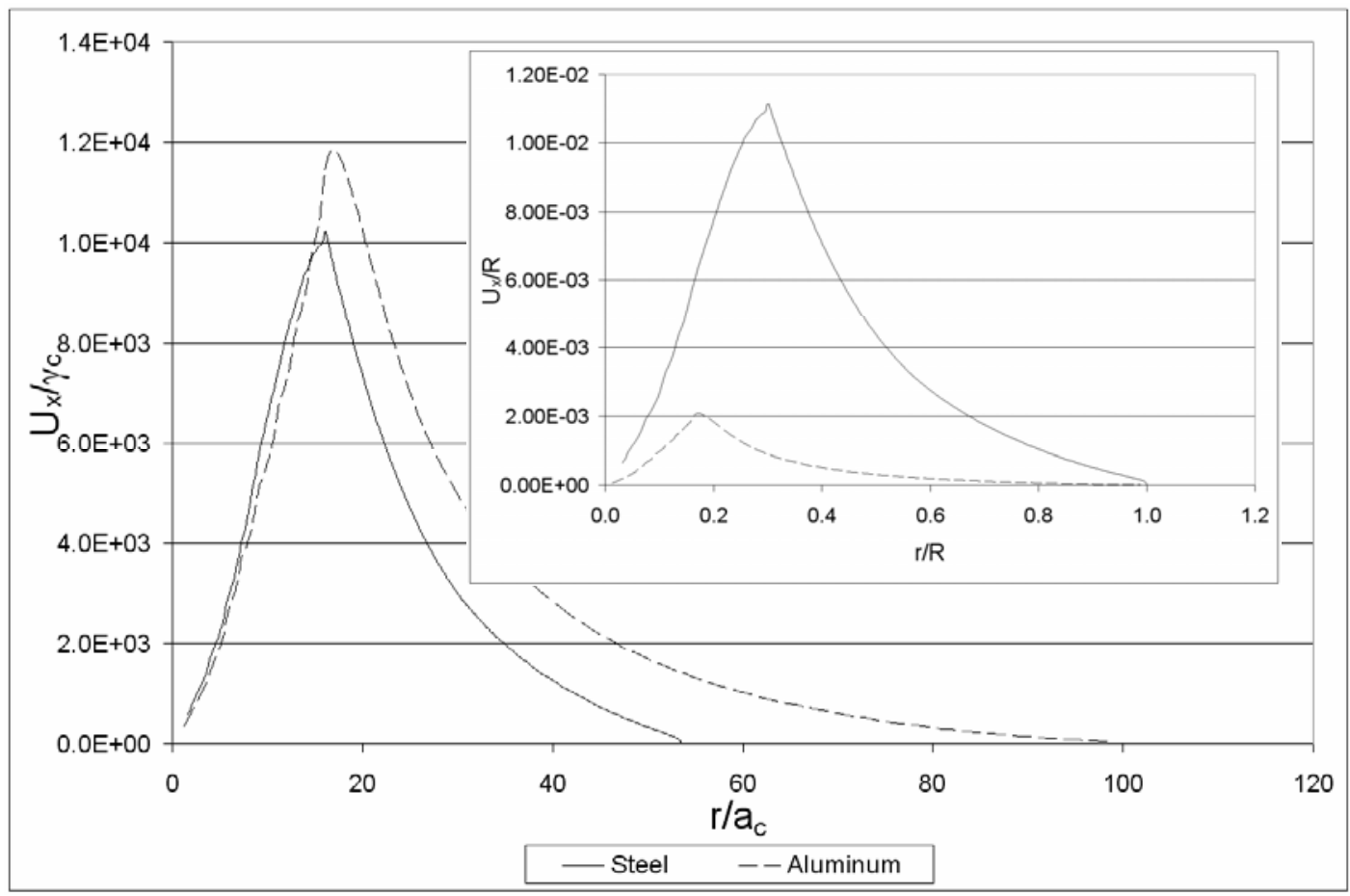

(a) Unloaded Radial Displacement

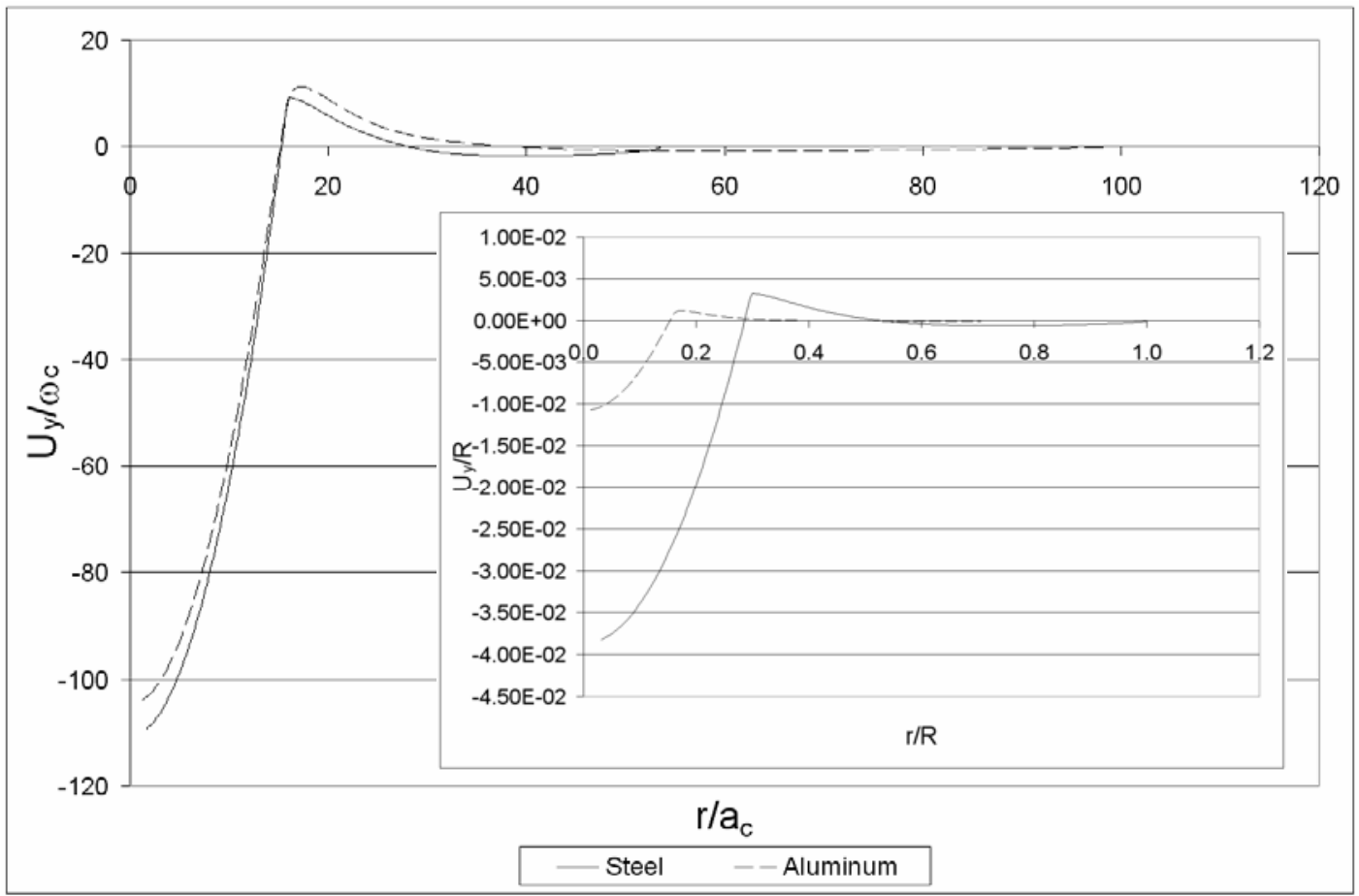

(b) Unloaded Axial Displacement

Figure 14: The normalized residual surface displacement of aluminum and steel hemispheres unloaded from $\omega^{*}=135$ versus the normalized radial location. 


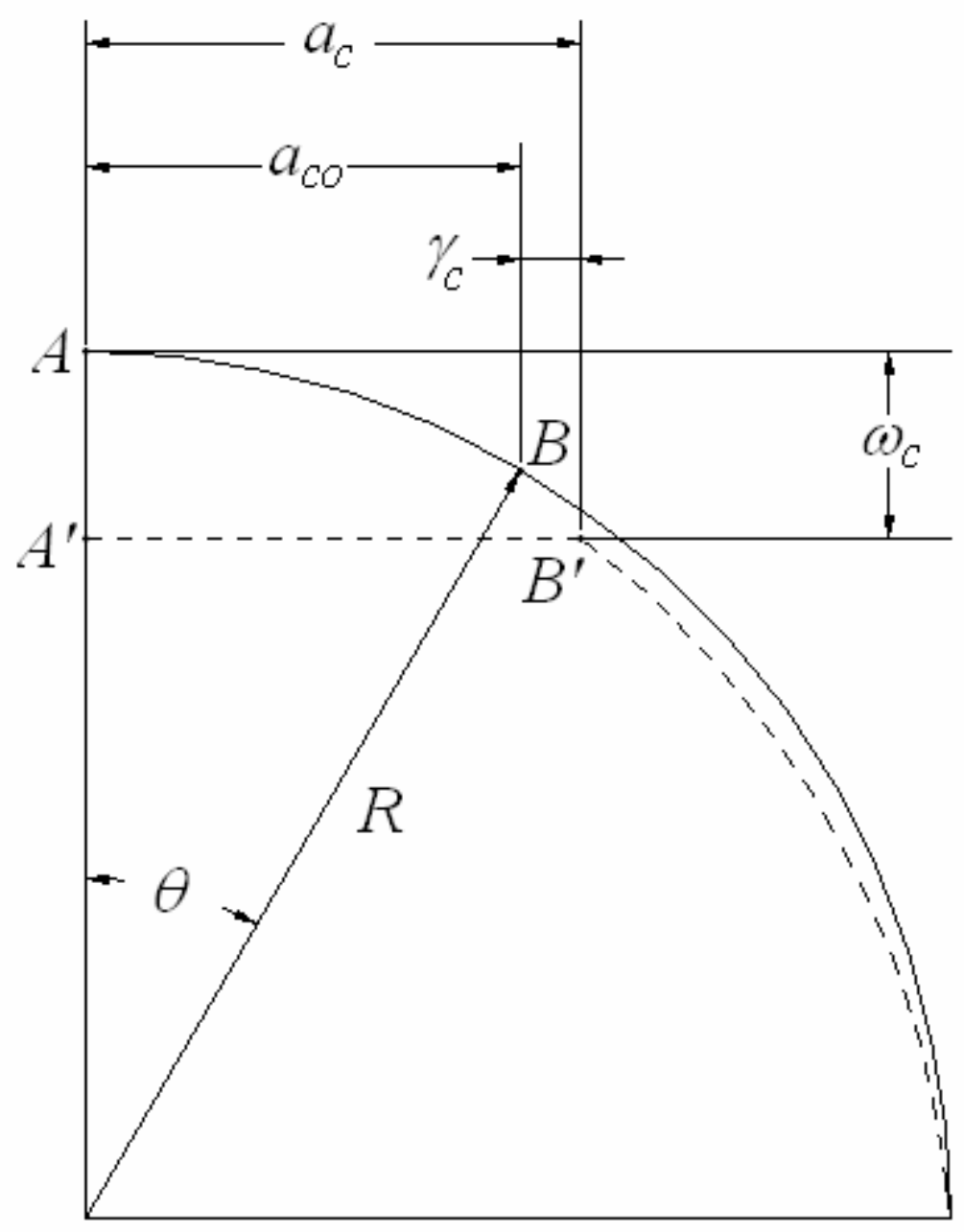

Figure 15: Schematic for the approximation of the location of the critical contact radius before loading (solid line) and after loading (dashed line). 